\title{
Microwave Dielectric Properties of Rocks
}

Fawwaz T. Ulaby, Tom Bengal, M. Craig Dobson, Jack East, Jim Garvin, and Diane Evans

\section{ABSTRACT}

A combination of several measurement techniques was used to investigate the dielectric properties of 80 rock samples in the microwave region. The real part of the dielectric constant, $\varepsilon^{\prime}$, was measured in $0.1 \mathrm{GHz}$ steps from $0.5 \mathrm{GHz}$ to $18 \mathrm{GHz}$, and the imaginary part, $\varepsilon^{\prime \prime}$, was measured at five frequencies extending between $1.6 \mathrm{GHz}$ and $16 \mathrm{GHz}$. In addition to the dielectric measurements, the bulk density was measured for all the samples and the bulk chemical composition was determined for 56 of the samples. This study shows that $\varepsilon^{\prime}$ is frequency-independent over the range of $0.5-18 \mathrm{GHz}$ for all rock samples, and that the bulk density $\rho_{\mathrm{b}}$ accounts for about $50 \%$ of the observed variance of $\varepsilon^{\prime}$. For silicate rocks, as much as $78 \%$ of the observed variance of $\varepsilon^{\prime}$ may be explained by the combination of density and the fractional contents of various oxides determined by $x$-ray fluorescence when the silicates are subgrouped by genesis (volcanic, plutonic and sedimentary). In contrast, the loss factor $\varepsilon "$ decreased with increasing frequency for most rock samples. It was not possible to establish"statistically significant relationships between $\varepsilon^{\prime \prime}$ and the measured density of the rock samples. However, in the case of silicate rocks, $60 \%$ of the variance in $\varepsilon$ " generally can be explained by bulk chemical composition when the silicates are subgrouped by genesis.

\footnotetext{
"F.T. Ulaby, Tom Bengal, M.C. Dobson, and J. East are with the Electrical Engineering and Computer Science Department, The University of Michigan, Ann Arbor, MI 48109

J. Garvin is with NASA Goddard Space Flight Center, Greenbelt, MD 20771

D. Evans is with the Jet Propulsion Laboratory, Pasadena, CA 91109
} 


\section{INTRODUCTION}

The purpose of this study is to investigate the microwave dielecric properties of igneous and sedimentary rock, in support of radar investigations of the Earth's geology and of the future radar and radiometer missions to Mars. Several studies have been reported in the literature on the dielectric properties of rocks [1-6], but in most of these studies the reported experimental measurements had been made either at $\mathrm{MHz}$ or lower frequencies, or at one or very few microwave frequencies. Thus, no continuous microwave spectra of the relative dielectric constant $\varepsilon$ have been reported to date. Furthermore, the majority of the reproted data for the dielectric loss factor $\varepsilon^{\prime \prime}$ is of questionable accuracy. This is because $\varepsilon^{\prime \prime}$ of most rocks is between 0.01 and 0.1 , and most dielectric measurement techniques do not have the accuracy required for measuring values that small.

The relative dielectric constant $\varepsilon$ of a material is defined as

$$
\varepsilon=\varepsilon^{\prime}-j \varepsilon^{\prime \prime},
$$

where the real part $\varepsilon^{\prime}$ is the perimittivity of the material (relative to that of free space) and the imaginary part $\varepsilon$ "is its dielectric loss factor (also relative to $\varepsilon_{0}$ of free space). This study focuses on the spectral region extending from $0.5 \mathrm{GHz}$ to $18 \mathrm{GHz}$. A combination of several measurement techniques was used to measure $\varepsilon$ over this frequency range. It included two probe techniques for measuring $\varepsilon^{\prime}$ in steps of 0.1 $\mathrm{GHz}$ from $0.5 \mathrm{GHz}$ to $18 \mathrm{GHz}$, and a resonant cavity perturbation technique for measuring $\varepsilon$ " at five frequencies extending from $1.6 \mathrm{GHz}$ to $16 \mathrm{GHz}$. Because these cavity measurements are very time-consuming, it was not possible to make the measurements at more than five frequencies.

The dielectric data reported in this study were generated from measurements performed for 80 rock samples. Each data point represents the average of several 
measurements corresponding to spatially different parts of the rock sample. The variability among measurements made for a given rock sample is an indicator of the sample's spatial inhomogeneity. Such variations may be due to density variations or to variations in chemical composition and crystalline structure among mineral constituents. In addition to the dielectric measurements, the bulk density was measured for all of the samples and the chemical composition was determined for 56 of the samples by $x$-ray fluorescence.

This paper provides statistical analyses relating $\varepsilon^{\prime}$ and $\varepsilon^{\prime \prime}$ of a rock sample to its density and chemical contents.

\section{DIELECTRIC PROBE MEASUREMENT TECHNIQUE}

The permittivity data reported in this study are based on measurements of the complex reflection coefficient of a coaxial probe terminated in the material under test. Two techniques were used. The first one is based on a third-order equivalent-circuit model that can be used for measuring the dielectric constant of any rock sample across the full frequency range of interest $(0.5-18 \mathrm{GHz})$. The second one is a simpler first-order equivalent-circuit model, but its validity range is limited to frequencies below $10 \mathrm{GHz}$ when $\varepsilon^{\prime}$ is larger than 8 . For all rock samples investigated in this study, $\varepsilon^{\prime}$ was found to be approximately independent of frequency over the $0.5-18 \mathrm{GHz}$ range.

Because it is simpler to use and calibrate, the first-order technique was initially used to measure $\varepsilon^{\prime}$ of a given sample, and if $\varepsilon^{\prime}$ was found to exceed 8 over the $0.5-10 \mathrm{GHz}$ range, the sample was remeasured using the more exact third-order technique. Brief descriptions of these two techniques are given next.

\subsection{Third-Order Equivalent Circuit}

The dielectric probe system (Fig. 1) consists of a swept RF source, a network analyzer (HP 8510A), and associated couplers and data processing instrumentation. Fig. 2(a) shows a cross-section of the probe tip and the dimensions of two of the 
probes examined in this study. The operation of open-ended coaxial lines to measure the dielectric constant of unknown materials is well-documented in the literature [7-10]. The input reflection coefficient at the probe tip, $\rho$, is given by

$$
\rho=\frac{Z_{L}-Z_{0}}{Z_{L}+Z_{0}}=\frac{Y_{0}-Y_{L}}{Y_{0}+Y_{L}},
$$

where $Y=1 / Z, Z_{0}$ is the line impedance, and $Z_{L}$ is the load impedance, which is governed by the geometry of the probe tip and the dielectric constant of the material it is in contact with or immersed in (for liquid materials). In general, an open-ended coaxial line may be described by an equivalent circuit of the form shown in Fig. 2(b). When placed in contact with a homogeneous material whose thickness is sufficient to simulate a slab of infinite electrical thickness, an open coaxial line has an admittance $Y_{L}(\omega, \varepsilon)$ given by

$$
Y_{L}(\omega, \varepsilon)=Y_{i}(\omega)+Y_{\theta}(\omega, \varepsilon)
$$

where $Y_{i}(\omega)=j \omega C_{i}$ is the "internal" admittance corresponding to the fringing capacitance $C_{i}$ that accounts for the fringing field in the Teflon region between the inner and outer conductors of the line. The "external" admittance $Y_{e}$, which is a function of both $\omega$ and the complex dielectric constant $\varepsilon$ of the material under test, consists of a frequency-dependent capacitor $C(\omega, \varepsilon)$ in parallel with a radiation conductance $G(\omega, \varepsilon)$

$$
Y_{\theta}(\omega, \varepsilon)=j \omega C(\omega, \varepsilon)+G(\omega, \varepsilon)
$$


The capacitor $C(\omega, \varepsilon)$ represents the fringing field concentration in the dielectric medium $(\varepsilon)$ surrounding the probe tip, and the conductance $G(\omega, \varepsilon)$ represents the radiation into the dielectric medium.

When the medium surrounding the probe tip is free space (i.e., an open-ended line), these two equivalent-circuit elements vary according to

$$
\begin{gathered}
C\left(\omega, \varepsilon_{0}\right)=C_{0}+B \omega^{2} \\
G\left(\omega, \varepsilon_{0}\right)=A \omega^{4},
\end{gathered}
$$

where $\mathrm{C}_{0}, \mathrm{~B}$, and $\mathrm{A}$ are constants for a given probe-tip geometry. If the radial dimensions of the coaxial line (namely, $r_{1}$ and $r_{2}$ ) are small compared to the wavelength $\lambda$, computations using the expressions given in Marcuvitz [11] yield values for $A$ and $B$ that are sufficiently small that the external admittance may be approximated as $Y_{e}\left(\omega, \varepsilon_{0}\right) \cong j \omega C_{0}$. If the dielectric constant of the medium surrounding the probe tip is not the free space value $\varepsilon_{0}$, however, the above simplification may lead to unacceptably large errors. Hence, in the general case we have

$$
Y_{\theta}\left(\omega, \varepsilon_{0}\right)=j \omega\left(C_{0}+B \omega^{2}\right)+A \omega^{4} .
$$

According to the theorem developed by Deschamps [12], the input admittance of an antenna immersed in a medium of complex dielectric constant $\varepsilon$ is related to the input admittance in free space through 


$$
Y_{\theta}(\omega, \varepsilon)=\sqrt{\frac{\varepsilon}{\varepsilon_{0}}} Y_{\theta}\left(\omega \sqrt{\frac{\varepsilon}{\varepsilon_{0}}}, \varepsilon_{0}\right)
$$

The above expression is for materials characterized by $\mu=\mu_{0}$. If we regard the openended coaxial line as an antenna and henceforth abbreviate the relative dielectric constant ratio $\varepsilon / \varepsilon_{0}$ as simply $\varepsilon$, we can write the following expression for the total input admittance of the probe when placed in contact with a material of relative dielectric constant $\varepsilon$.

$$
Y_{L}(\omega, \varepsilon)=j \omega C_{i}+j \omega C_{0} \varepsilon+j B \omega^{3} \varepsilon^{2}+A \omega^{4} \varepsilon^{25}
$$

With the line admittance $Y_{0}$ known, measurements of the amplitude and phase of $\rho$ by the network analyzer system (Fig. 1) lead to a measurement of $Y_{L}$. The next step is to determine $\varepsilon$ from $Y_{L}$. This is accomplished by (1) calibrating the measurement probe in order to establish the values of the constants $C_{i}, C_{0}, B$, and $A$, and (2) developing an iterative program for finding a value for $\varepsilon$ that minimizes the error between the measured value of $Y_{L}$ and the value calcuated from the expression on the right-hand side of (8).

Calibration entails finding the values of the constants $C_{i}, C_{0}, B$, and $A$ of $(8)$ for each probe used in this study. Under ideal circumstances, one needs to determine these constants only once and at only one frequency. The equivalent-circuit model, however, is only approximate; hence, it is necessary to determine these constants at each frequency that the probe is intended to be used. Each dielectric probe was calibrated by measuring the complex reflection coefficient under four termination conditions: (1) short circuit, (2) open circuit, (3) probe immersed in distilled water, and 
(4) probe immersed in methanol. Distilled water and methanol were used because their dispersion spectra are well known [13], [14].

\subsection{First-Order Equivalent Circuit}

If the diameter of the coaxial probe is much smaller than the wavelength in the material under test, the expression given by (6) and (7) for the equivalent-circuit admittance $Y_{e}(\omega, \varepsilon)$ simplifies to only one term, $j \omega C_{0} \varepsilon$, because the other two terms become negligibly small. For the 0.14 -in probe used in this study, the conditoin

$$
f(G H z) \leq 50 / \sqrt{\varepsilon^{\prime}},
$$

must be satisfied in order for the first-order model to yield accurate results. This condition was found by comparing measurements made with this technique to measurements made using the more-exact technique described in the previous section.

\subsubsection{Reflection Measurement Technique}

For the first-order equivalent circuit, the admittance,

$$
Y_{L}(\omega, \varepsilon) \cong j \omega C_{i}+j \omega C_{0} \varepsilon
$$

can be determined by measuring the reflection coefficient $\rho$. The constants $C_{i}$ and $C_{0}$ can be determined by measuring $Y_{L}(\omega, \varepsilon)$ for two materials with known $\varepsilon$. With the constants known, $\varepsilon$ of an unknown material may be computed directly from 


$$
\varepsilon=\frac{1}{C_{0}}\left[\frac{Y_{0}}{j \omega}\left(\frac{1-\rho}{1+\rho}\right)-C_{i}\right]
$$

by measuring $\rho$. The coaxial line is a standard 50 -ohm line (i.e., $Y_{0}=1 / 50$ ).

\subsubsection{Group-Delay Measurement Technique}

As an alternative to measuring $\rho$ (in order to determine $\varepsilon$ ), a group-delay technique was developed which requires calibration against only one calibration material rather than two. For low loss materials with $\varepsilon^{\prime \prime}<<\varepsilon^{\prime}$,

$$
Y_{L} \cong j \omega\left(C_{i}+\varepsilon^{\prime} C_{0}\right)
$$

and the reflection coefficient

$$
\begin{aligned}
\rho & =|\rho| e^{j \phi} \\
& =\frac{Y_{0}-Y_{L}}{Y_{0}+Y_{L}}
\end{aligned}
$$

has a phase angle given by

$$
\phi=2 \cot ^{-1}\left[50 \omega\left(C_{i}+\varepsilon^{\prime} C_{0}\right)\right]
$$

For the 0.141 -in probe, the constants $C_{i}$ and $C_{0}$ are on the order of 0.02 picofarads. Consequently, the entire quantity inside the square brackets is much smaller than 1 if $f \leq 20 \mathrm{GHz}$ and $\varepsilon^{\prime} \leq 10$. Hence, $\cot ^{-1}$ () may be expanded in a Taylor series 


$$
\cot ^{-1}(x)=\pi / 2-x+x^{3} / 3-\ldots,
$$

and if we retain only the first two terms, we have

$$
\phi \cong \pi-100 \omega\left(C_{i}+\varepsilon^{\prime} C_{0}\right)
$$

The group delay $\tau$ is defined as the change in the phase $\phi$ with angular frequency,

$$
\tau=\frac{\partial \phi}{\partial \omega}=-100\left(C_{i}+\varepsilon^{\prime} C_{0}\right) .
$$

If the group delay is measured with the probe in air (with $\varepsilon^{\prime}=1$ ) and not in contact with any other material, we get the reference group delay $\tau_{0}$,

$$
\tau_{0}=-100\left(C_{i}+C_{0}\right)
$$

The differential group delay is defined as

$$
\begin{aligned}
\Delta \tau & =\tau-\tau_{0} \\
& =-100 C_{0}\left(\varepsilon^{\prime}-1\right),
\end{aligned}
$$

from which we obtain the expression

$$
\varepsilon^{\prime}=1-\frac{\Delta \tau}{100 C_{0}}
$$


The constant $C_{0}$ may be determined by measuring $\Delta \tau$ for one material of known $\varepsilon^{\prime}$. The group delays $\tau$ and $\tau_{0}$ can be measured directly by the HP 8510A network analyzer.

Comparison of results using the group-delay technique with results obtained using the more-exact reflection coefficient technique has led to the conclusion that the condition

$$
f(\mathrm{GHz}) \leq 30 / \sqrt{\varepsilon^{\prime}},
$$

should be satisfied in order for the approximation made in going from (15) to (17) to be valid.

\subsection{Sample Preparation For Permittivity Measurements}

When using the coaxial probe to measure the permittivity of a solid material, the following two conditions must be satisfied (in order for the measurements to produce accurate results):

(1) The thickness of the sample must be at least equal to the probe diameter. For the 0.14 -in probe, this condition is satisfied if the thickness is greater than $4 \mathrm{~mm}$.

(2) The surface of the sample in contact with the probe must be very smooth in order to insure good electrical contact. This was achieved by having each rock sample cut with a rock saw to obtain a flat surface and then the surface was smoothed using a table-top rotary sander.

To avoid dielectric effects that may be caused by the possible presence of surficial water molecules on the sample, each sample was dried in an oven for 15 minutes at $105^{\circ} \mathrm{C}$ prior to performing the dielectric measurements. It was found, 
however, that there was very little difference in $\varepsilon^{\prime}$, if any, between the results obtained after drying the samples and those obtained on the basis of the measurements made prior to drying the samples. An entirely different conclusion was reached for the measurements of the dielectric loss factor $\varepsilon^{\prime \prime}$; for some rocks, the values measured prior to drying the sample were as much as twice the values measured for the samples after drying.

\subsection{Measurement Accuracy and Precision}

The measurement accuracy of the probe technique was evaluated by comparing the permittivity measured by the probe with the permittivity of standard materials. The reference materials are homogeneous, thick blocks of solid materials, such as teflon, whose dielectric constants had been carefully measured using waveguide techniques. Based on such comparisons, the probe measurement accuracy was found to be better than \pm 0.03 of the measured value.

By accuracy, we refer to the absolute level of $\varepsilon^{\prime}$, whereas by precision, we refer to the variability associated with the spatial inhomogeneity of the sample. For all rock samples, $\varepsilon^{\prime}$ was measured by applying the probe to at least 16 spatially different locations on the polished surface of the rock sample. In each case, we computed the mean value of $\varepsilon^{\prime}$, the associated standard deviation $s$ and the ratio $s / \varepsilon^{\prime}$. For all samples, the ratio $s / \varepsilon^{\prime}$ was found to be smaller than 0.12 , and for most the ratio was smaller than 0.05 .

Figure 3 shows typical permittivity spectra of four rock samples. The absence of a discernible dependence on frequency was characterisitic of all samples measured in this study and is in agreement with previous conclusions reached by Olhoeft et al. [2]. Hence, in all forthcoming discussions and analyses, $\varepsilon^{\prime}$ will be treated as frequency independent and will be represented by the average value measured over the $0.5-18$ $\mathrm{GHz}$ range. 


\section{RESONANT-CAVITY PERTURBATION TECHNIQUE}

A resonant cavity is a closed volume. The diagram in Fig. 4(a) shows a cylindrical cavity with two magnetic loop couplers protruding slightly into the cavity volume on the inside walls and connected to SMA connectors on the outside walls at a height midway between the top side (the lid) and the cavity floor. Figure 5 shows the measurement system.

With the cavity empty, if one were to connect a signal generator (HP 8350B in Fig. 5) to one of the connectors and a network analyzer to the other and then sweep the generator frequency across the resonance region of the cavity, the output power would be a Gaussian-like function of frequency (Fig. 4(b)). This power spectrum is characterized by $f_{0}$, the frequency at which the power is a maximum, and by $Q_{0}$, the quality factor,

$$
Q_{0}=\frac{f_{0}}{\Delta f},
$$

where $\Delta f$ is the half-power width of the power spectrum. If we insert a dielectric material into the cavity, the spectrum will change in two ways: (1) the resonant frequency decreases to a lower value, which we shall call $f_{s}$, and $(2)$ the quality factor decreases to a lower value $Q_{S}$.

In order to maintain $Q_{S}$ large (i.e., maintain a resonant-like spectrum), the volume of the material inserted into the cavity must be kept small relative to the cavity volume. When this is the case, the resonant-cavity perturbation technique [15] may be used to determine $\varepsilon^{\prime}$ and $\varepsilon^{\prime \prime}$ of a dielectric material from measurement of $f_{0}, f_{S}, Q_{0}$, and Qs. 
For a cylindrical cavity with radius a and height $d<2 a$ oscillating in the $\mathrm{TM}_{010}$ mode and containing a needle-shaped dielectric material oriented along the vertical axis of the cylinder, the shift in the resonant frequency is

$$
\frac{f_{s}-f_{0}}{f_{s}}=-1.855 V\left(\varepsilon^{\prime}-1\right)
$$

if the volume fraction of the cavity occupied by the sample is small. Solving for $\varepsilon^{\prime}$ we get

$$
\varepsilon^{\prime}=1+\frac{f_{0}-f_{s}}{1.855 f_{s} V}
$$

If the material has a dielectric loss factor $\varepsilon^{\prime \prime}$, it can be shown $[14, p .373]$ that

$$
\varepsilon^{\prime \prime} \cong \frac{\left(\varepsilon^{\prime}-1\right)}{2}\left(\frac{f_{0}}{f_{0}-f_{s}}\right)\left(\frac{1}{Q_{s}}-\frac{1}{Q_{0}}\right)
$$

These expressions are valid only if $V$ is very small, and (24) is valid only if the dielectric material is approximately needle shaped and oriented vertically. One of the major problems associated with using this method to determine $\varepsilon^{\prime}$ and $\varepsilon^{\prime \prime}$ is the need to know $\vee$ very accurately $\left(\varepsilon^{\prime \prime}\right.$ depends on $\left(\varepsilon^{\prime}-1\right)$ which, in turn, depends directly on $1 / \mathrm{V})$. In our case, however, we did not need to know $V$ exactly becuase we already know $\varepsilon^{\prime}$ from the probe measurements discussed in the previous section. Hence, $\varepsilon^{\prime \prime}$ could be determined from (26) without the need to measure V. This procedure of using dielectric probes to measure $\varepsilon^{\prime}$ and resonant cavities to measure $\varepsilon^{\prime \prime}$ proved extremely effective because the errors associated with the handling and the 
measuring of the weight and volume of very small rock samples are intolerably high. As will be discussed below, a desirable value for $V$ is about 0.5 percent. For a cavity volume of $2.5 \mathrm{~cm}^{3}$ (which was the volume of one of the cavities used in this study), $V$ would have to be about $1.25 \times 10^{-2} \mathrm{~cm}^{3}$ and the corresponding weight would be about $31 \mathrm{mg}$ (for a typical density of $2.5 \mathrm{~g} / \mathrm{cm}^{3}$ ).

\subsection{Measurement Accuracy and Presicion}

By way of evaluating the measurement technique as well as establishing the range of validity of (26) as a function of $\mathrm{V}$, we conducted a carefully designed experiment in which $\varepsilon^{\prime \prime}$ of plexiglass was measured as a function of $V$ for values of $V$ extending from $10^{-3}$ percent to 10.1 percent. We chose plexiglass because its complex dielectric constant is well known $(\varepsilon=2.55-\mathrm{j} 0.0165)$ and its dielectric loss factor is small. The results of the experiment are shown in Fig. 6 . The measurement technique predicted the correct value for $\varepsilon^{\prime \prime}$ within an $r m s$ error of 0.001 for the range 0.01 percent $\leq V \leq 1$ percent, and with a slightly larger error for $V$ up to 5 percent. $A$ detailed anlaysis of the errors associated with the measurements of the quantities $f_{0}$, $f_{S}, Q_{0}$, and $Q_{S}$ led to the conclusion that the optimum range of $V$ is between 0.5 percent and 1 percent, and that if $V$ is in this range the minimum measurable value of $\varepsilon$ " is around 0.002 . For each rock sample, the $\varepsilon^{\prime \prime}$ data reported in this study are averages of measurements conducted for five small sub-samples of the (parent) sample.

For a few of the measured samples, $\varepsilon$ " was observed to exhibit no discernible dependence on frequency. For most samples, however, $\varepsilon^{\prime \prime}$ decreased with increasing frequency over the $1.6 \mathrm{GHz}-16 \mathrm{GHz}$ range. Typical examples of these two types of spectra are shown in Fig. 7.

\subsection{Cavity Characteristics}


Five cylindrical cavities were used in this study, with center frequencies ranging from $1.6 \mathrm{GHz}$ to $16 \mathrm{GHz}$. Table 1 provides a listing of their pertinent characteristics. Of particular note is the cavity volume, ranging from approximately $1000 \mathrm{~cm}^{3}$ for the 1.6 $\mathrm{GHz}$ cavity to only $1 \mathrm{~cm}^{3}$ for the $16 \mathrm{GHz}$ cavity.

\section{MEASURED DATA}

The measured data has been tabulated in a technical report [16]. For each sample, the entries include (1) rock type, (2) rock \# and source, (3) density, (4) $\varepsilon^{\prime}$ (average value over the $0.5-18 \mathrm{GHz}$ range), (5) $\mathrm{s} / \varepsilon^{\prime}$, the standard derivation-to-mean ratio of the measured value of $\varepsilon^{\prime},(6) \varepsilon^{\prime \prime}$ at $1.6,5.0,7.8,11.4$, and $16.0 \mathrm{GHz}$, and (7) the weight fractions of 10 chemical compounds (for only 56 of the 80 rock samples). The information on chemical composition was obtained using $x$-ray fluorescence. The distribution of the 80 samples by rock type is given in conjunction with Fig. 8 .

\section{ANALYSIS OF PERMITTIVITY DATA}

Among the 80 rock samples, the measured value of $\varepsilon^{\prime}$ ranged between 2.5 and 8.3. The ranges for individual rock groups are presented in a horizontal bar-chart format in Fig. 8.

\subsection{Dependence of $\varepsilon^{\prime}$ on Density}

According to previous studies [1-6], the density $\rho$ of the rock is the single most important parameter governing the magnitude of $\varepsilon^{\prime}$. One of the commonly used formulas relating $\varepsilon$ to $\rho$ is based upon the geometric mean formula where

$$
\log \varepsilon=\sum_{i} V_{i} \log \varepsilon_{i}
$$


where $V_{i}$ is the volume fraction of the $i^{\text {th }}$ component of the material and is related to density by

$$
V_{i}=\rho_{b} / \rho_{s}
$$

where $\rho_{\mathrm{b}}$ and $\rho_{\mathrm{s}}$ are the bulk and specific densities of the rock material, respectively. Thus, a two component mixture of solid rock and air is described by

$$
\log \varepsilon_{\rho_{b}}^{\prime}=\frac{\rho_{b}}{\rho_{s}} \log \varepsilon_{\rho_{s}}^{\prime}
$$

Hence, at an arbitrary density $\rho$

$$
\varepsilon_{\rho_{b}}^{\prime}=\varepsilon_{\rho}^{\rho_{p} / \rho}
$$

Campbell and Ulrichs [1] conducted measurements for a large number of powdered rocks, all at a density of $\rho_{b}=1 \mathrm{~g} / \mathrm{cm}^{3}$, and found that $\varepsilon^{\prime} \rho_{b}$ varied over the narrow range between 1.9 and 2.1 for most of the 25 different types of powdered rocks measured and that the mean value is around 2.0. Upon setting $\rho=1 \mathrm{~g} / \mathrm{cm}^{3}$ and $\varepsilon_{\rho}^{\prime}=2$ in (30), we find $\varepsilon_{\rho_{b}}^{\prime}=2^{\rho_{b}}$. This result is in close agreement with the formula used by Olhoeft and Strangway [4] who found $\varepsilon_{\rho_{b}}^{\prime}=(1.93 \pm 0.17)^{\rho_{b}}$ in their analysis of moon rocks. For the data measured in the present study, application of the geometric mean formula yields a minimum mean-square error if $\varepsilon_{\rho}^{\prime}$ is set equal to $1.96 \pm 0.14$;

$$
\varepsilon_{\rho_{b}}^{\prime}=(1.96 \pm 0.14)^{\rho_{b}}
$$


This function is shown in Figure 9 along with the measured data. The linear correlation coefficient between the values predicted by (31) and the measured values of $\varepsilon^{\prime}$ is $R=0.72$. Hence, about $50 \%$ of the variance in the data can be attributed to the density of the rock samples; the remaining data scatter about the regression curve is attributed to dependence of $\varepsilon^{\prime}$ on the mineral composition of the rocks.

The variation of $\varepsilon^{\prime}$ with $\rho_{b}$ is also shown in Figure 9 for individual rock types. The carbonates exhibit the narrowest density range, followed in order by igneous, volcanic and sedimentary silicates. The igneous volcanic silicates are found to have the strongest sensitivity of $\varepsilon^{\prime}$ to $\rho_{b}$.

\subsection{Dependence of $\varepsilon^{\prime}$ on Chemical Composition}

The geometric mean formulation given by (27) can be used to investigate the dependence of $\varepsilon^{\prime}$ on bulk mineral composition of rock. Ideally, the volume fraction $V_{i}$ of each discrete constituent mineral would be known for use in the analysis. However, the rock chemistry of the samples used in the present study is ascertained by $x$-ray fluorescence which provides the mass percent $W_{i}$ of various oxides for the bulk material. Consequently, the following analysis evaluates the dependence of $\varepsilon^{\prime}$ on bulk chemical composition.

$X$-ray fluorescence of the silicate samples yields values for the mass percent $W_{i}$ of 10 oxides plus the percent loss on ignition of the sample (LOI). The volume fraction of each oxide can be approximated by

$$
V_{i}=\rho_{b} W_{i} / M_{i}
$$


where $M_{i}$ is the molecular weight of the $i^{\text {th }}$ oxide constituent. Hence, for the ten measured oxides plus loss on ignition (LOI)

$$
\begin{aligned}
\log \varepsilon^{\prime} & =\sum_{i=1}^{11} \frac{\rho_{b} W_{i}}{M_{i}} \log \varepsilon_{i}^{\prime} \\
& =\rho_{b} \sum_{i=1}^{11} \frac{W_{i}}{M_{i}} \log \varepsilon_{i}^{\prime}
\end{aligned}
$$

An empirical evaluation of the dependence of $\varepsilon^{\prime}$ on bulk chemical composition is possible for the silicate rock samples which had been measured by $x$-ray fluorescence. A multiple linear regression analysis of the form given in (33) utilized measurements of $\varepsilon^{\prime}, \rho_{b}$ and $W_{i} / M_{i}$ to yield estimates of $\log \varepsilon^{\prime}$. In order to gain some understanding of the role of mineralogy and crystal size and structure in determining $\varepsilon^{\prime}$, the analysis is conducted for all silicate rocks treated as a single class and as subdivided by genesis into volcanic, plutonic and sedimentary subgroups. Since $x$-ray fluorescence provides measurements of 10 oxides plus loss on ignition (LOI) and the smallest subclass, plutonic silicates, contains only 14 samples, a stepwise approach is used in the analysis wherein at each step an oxide or (LOI) is added to the regression on the basis of its F-ratio. In this analysis it is presumed that LOI is dominated by the liberation of bound water during sample ignition and hence the molecular weight of $\mathrm{H}_{2} \mathrm{O}$ is assumed.

For all silicate rock samples combined (54 samples), the regression analysis is truncated after the inclusion of 6 variables with 8.44 as the $F$ to enter of the 6 th variable and a resultant standard error of the estimate (SEE) of 0.025 . The regression equation is 


$$
\begin{aligned}
\log \varepsilon^{\prime}=\rho_{\mathrm{b}} & \frac{.131( \pm .007) \mathrm{W}_{\mathrm{Si}_{2}}}{\mathrm{M}_{\mathrm{Si}_{2}}}+\frac{.637( \pm .062) \mathrm{W}_{\mathrm{Al}_{2} \mathrm{O}_{3}}}{\mathrm{M}_{\mathrm{Al}_{2} \mathrm{O}_{3}}} \\
& +\frac{.517( \pm .093) \mathrm{W}_{\mathrm{Fe}_{2} \mathrm{O}_{3}}}{\mathrm{M}_{\mathrm{Fe}_{2} \mathrm{O}_{3}}}+\frac{.11( \pm .031) \mathrm{W}_{\mathrm{LOI}}}{\mathrm{M}_{\mathrm{LOI}}} \\
& \left.+\frac{.1( \pm .021) \mathrm{W}_{\mathrm{M}_{\mathrm{g}} \mathrm{O}}}{\mathrm{M}_{\mathrm{Mg}_{\mathrm{O}}}}+\frac{.626( \pm .216) \mathrm{W}_{\mathrm{K}_{2} \mathrm{O}}}{\mathrm{M}_{\mathrm{K}_{2} \mathrm{O}}}\right)
\end{aligned}
$$

The variables in (34) are listed in decreasing order of significance. The estimates of $\varepsilon^{\prime}$ provided by (34) are related to the measured values of $\varepsilon^{\prime}$ by

$$
\varepsilon^{\prime}=0.643+0.891 \varepsilon_{\text {calculated }}^{\prime}
$$

with a linear correlation coefficient of $r=0.835$ for the 54 silicate samples.

For each silicate subclass, the regression analysis is truncated after inclusion of four variables and yields:

a) volcanic silicate rocks, 20 samples, $\mathrm{SEE}=.02$,

$$
\begin{aligned}
\log \varepsilon^{\prime}=\rho_{\mathrm{b}} & \left(\frac{.174( \pm .01) \mathrm{W}_{\mathrm{Si}_{2}}}{\mathrm{M}_{\mathrm{Si}_{2}}}+\frac{.62( \pm .06) \mathrm{W}_{\mathrm{Ca} 0}}{\mathrm{M}_{\mathrm{Ca} 0}}\right. \\
+ & \left.\frac{1.334( \pm .256) \mathrm{W}_{\mathrm{K}_{2} \mathrm{O}}}{\mathrm{M}_{\mathrm{K}_{2} \mathrm{O}}}+\frac{14.808( \pm 5.364) \mathrm{W}_{\mathrm{PO}_{5}}}{\mathrm{M}_{\mathrm{PO}_{5}}}\right)
\end{aligned}
$$


b) plutonic silicate rocks, 14 samples, SEE $=.02$

$$
\begin{aligned}
\log \varepsilon^{\prime}=\rho_{\mathrm{b}} & \left(\frac{.165( \pm .011) \mathrm{W}_{\mathrm{Si}_{2}}}{\mathrm{M}_{\mathrm{Si}_{2}}}+\frac{1.995( \pm .197) \mathrm{W}_{\mathrm{Fe}_{2} \mathrm{O}_{3}}}{\mathrm{M}_{\mathrm{Fe}_{2} \mathrm{O}_{3}}}\right. \\
& \left.+\frac{.855( \pm .139) \mathrm{W}_{\mathrm{Na}_{2} \mathrm{O}}}{\mathrm{M}_{\mathrm{Na}_{2} \mathrm{O}}}-\frac{1.687( \pm .439) \mathrm{W}_{\mathrm{T}_{\mathrm{i}} \mathrm{O}_{2}}}{\mathrm{M}_{\mathrm{Ti}_{2}}}\right)
\end{aligned}
$$

c) sedimentary silicate rocks, 20 samples, SEE $=.028$,

$$
\begin{aligned}
\log \varepsilon^{\prime}=\rho_{\mathrm{b}} & \left(\frac{.111( \pm .016) W_{\mathrm{Fe}_{2} \mathrm{O}_{3}}}{\mathrm{M}_{\mathrm{Fe}_{2} \mathrm{O}_{3}}}+\frac{.982( \pm .353) \mathrm{W}_{\mathrm{Si}_{2}}}{\mathrm{M}_{\mathrm{Si}_{2}}}\right. \\
& \left.+\frac{.157( \pm .045) \mathrm{W}_{\mathrm{Ti}_{2}}}{\mathrm{M}_{\mathrm{Ti}_{2}}}+\frac{2.561( \pm .677) \mathrm{W}_{\mathrm{Na}_{2} \mathrm{O}}}{\mathrm{M}_{\mathrm{Na}_{2} \mathrm{O}}}\right)
\end{aligned}
$$

The values of $\varepsilon^{\prime}$ estimated by (35) to (37) are related to the measured values of $\varepsilon^{\prime}$ by

$$
\varepsilon^{\prime}=0.864+0.841 \varepsilon_{\text {calculated }}^{\prime}
$$

with a linear correlation coefficient of $r=0.886$ as shown in Figure 10 . The most significant oxides are found to be $\mathrm{Si}_{2}$ and $\mathrm{Fe}_{2} \mathrm{O}_{3}$. However, the variability in the oxides selected for each class and the differences of their respective regression coefficients between subclasses indicates that bulk chemical composition and density alone are not accounting for all of the observed sample variance in a robust fashion. 
This implies that mineralogy per se and crystalline structure also exert a strong residual influence on $\varepsilon^{\prime}$.

The significance of mineral composition is illustrated by a sample of gabbro in Figure 11. The average value of $\varepsilon^{\prime}$ for this sample is 6.6. However, olivine inclusions are found to have much larger values of $\varepsilon^{\prime}$ ranging from 7.2 to 13.2.

\subsection{Dependence of Dielectric Loss on Density and Chemical}

\section{Composition}

The measured dielectric loss factor $\varepsilon^{\prime \prime}$ ranged between $<0.002$ to 0.245 as shown in Figure 12 and, unlike $\varepsilon^{\prime}$, the loss factor is found to generally decrease with frequency. Prior to measurement in the resonant cavities, all samples were oven dried for 15 minutes at $105^{\circ} \mathrm{C}$ in order to liberate potential layers of moisture absorbed from handling or from the laboratory atmosphere. The effects of this drying process on $\varepsilon^{\prime \prime}$ are shown in Figure 13 for samples of basalt and siltstone. In general, the drying process had little effect upon $\varepsilon^{\prime \prime}$ of igneous samples and more pronounced effect for sedimentary samples. However, even with the low temperature drying (at $105^{\circ} \mathrm{C}$ ), the vast majority of rock samples display a decrease of $\varepsilon^{\prime \prime}$ with frequency. This is illustrated by Figure 14 which shows the average loss of 72 rock samples as a function of frequency. This behavior may be partially attributable to the effects of bound water within the rock samples. The frequency behavior of the average dielectric loss factor for each gereralized rock type is shown in Figure 15. $\varepsilon^{\prime \prime}$ is the lowest for carbonates and the highest for volcanic silicates. The decrease of $\varepsilon^{\prime \prime}$ with frequency is found to be the greatest for sedimentary and plutonic silicates.

The loss factor $\varepsilon^{\prime \prime}$ may be modeled as the sum of a conductive component $\varepsilon_{c}{ }^{\prime \prime}$ and a frequency-independent residual component $\varepsilon_{r}$ ", 


$$
\begin{aligned}
\varepsilon^{\prime \prime}(f) & =\varepsilon_{r}^{\prime \prime}+\varepsilon_{c}^{\prime \prime}(f) \\
& =\varepsilon_{r}^{\prime \prime}+\frac{\sigma}{2 \pi f \varepsilon_{0}} \\
& \triangleq P_{1}+P_{2} / f
\end{aligned}
$$

where $\sigma$ is the conductivity, and $P_{1}$ and $P_{2}$ are abbreviations for $\varepsilon_{r}{ }^{\prime \prime}$ and $\sigma / 2 \pi \varepsilon_{0}$, respectively. For each of the measured rock samples, the values of $P_{1}$ and $P_{2}$ are determined by fitting the data obtained at five frequencies $(1.6,5.0,7.8,11.4$, and 16.0 $\mathrm{GHz}$ ) to the linear function given by (38). Figure 16 presents the values of $P_{1}$ and $P_{2}$ determined for each of the rock samples. The constant $P_{2}$ of a rock sample is proportional to its conductivity $\sigma$ and is given in units of $\mathrm{GHz}^{-1}$. The values of $\mathrm{P}_{1}$ and $\mathrm{P}_{2}$ are shown be be uncorrelated.

Unlike $\varepsilon^{\prime}$, which exhibits a strong dependence on bulk density, the loss factor $\varepsilon^{\prime \prime}$ is not significantly correlated with $\rho_{b}$ at any of the measured frequencies. This same conclusion applies to $P_{1}$ and $P_{2}$ as shown in Figure 17.

The role of bulk chemical composition in the determination of the loss factor is evaluated in a fashion similar to that presented for $\varepsilon^{\prime}$. The conductive and frequencyindenendent components of $\varepsilon^{\prime \prime}$ are each empirically related to the bulk chemistry by multiple linear regression analyses on $\mathrm{P}_{1}$ and $\mathrm{P}_{2}$ using a stepwise approach,

$$
\begin{aligned}
& \mathrm{P}_{1}=\sum_{i} \mathrm{~V}_{\mathrm{i}} \mathrm{P}_{1 \mathrm{i}, \mathrm{g}} \\
& \mathrm{P}_{2}=\sum_{\mathrm{i}} \mathrm{V}_{\mathrm{i}} \mathrm{P}_{2 \mathrm{i}, \mathrm{g}}
\end{aligned}
$$


where $V_{i}=\rho_{b} W_{i} / M_{i}$ in moles $/ \mathrm{cm}^{3}$ and the subscripts $i$ and $g$ refer to the specific oxide (from $x$-ray diffraction analysis) and rock sub-category, respectively.

For the frequency-independent component $P_{1}$ of the loss factor, the regression results for treatment of all 54 silicate rock samples as a single class yield

$$
\begin{aligned}
& P_{1}=\rho_{b}\left(\frac{.19( \pm .114) W_{\mathrm{Fe}_{2} \mathrm{O}_{3}}}{\mathrm{M}_{\mathrm{Fe}_{2} \mathrm{O}_{3}}}+\frac{.082( \pm .033) \mathrm{W}_{\mathrm{Al}_{2} \mathrm{O}_{3}}}{\mathrm{M}_{\mathrm{Al}_{2} \mathrm{O}_{3}}}\right. \\
& \left.+\frac{.051( \pm .018) W_{\text {LOI }}}{M_{\text {LOI }}}-\frac{1.132( \pm .806) W_{M_{n} 0}}{M_{M_{n} 0}}\right)
\end{aligned}
$$

with SEE $=.019$. The estimates of $P_{1}$ provided by (41) are found to be very poorly correlated with the values of $P_{1}$ determined for each of 54 samples $(r=.42)$ and with a regression

$$
P_{1}=0.007+0.86 P_{1 \text { calculated }}
$$

Consideration of each silicate subclass seperately yields

a) volcanic silicate rocks, 20 samples, SEE $=.015$

$$
\begin{gathered}
P_{1}=\rho_{b}\left(\frac{1.563( \pm .434) W_{\mathrm{Fe}_{2} O_{3}}}{M_{\mathrm{Fe}_{2} \mathrm{O}_{3}}}-\frac{.232( \pm .107) \mathrm{W}_{\mathrm{Ca} 0}}{\mathrm{M}_{\mathrm{Ca} 0}}\right. \\
\left.-\frac{.167( \pm .099) \mathrm{W}_{\mathrm{M}_{\mathrm{g}} \mathrm{O}}}{\mathrm{M}_{\mathrm{Mg}_{\mathrm{g}}}}+\frac{.186( \pm .067) \mathrm{W}_{\mathrm{LOI}}}{\mathrm{M}_{\mathrm{LOI}}}\right)
\end{gathered}
$$


b) plutonic silicate rocks, 14 samples, SEE $=.004$

$$
\begin{aligned}
P_{1}=\rho_{b} & \left(\frac{.62( \pm .076) W_{\mathrm{Fe}_{2} \mathrm{O}_{3}}}{\mathrm{M}_{\mathrm{Fe}_{2} \mathrm{O}_{3}}}-\frac{.465( \pm .105) \mathrm{W}_{\mathrm{T}: 0_{2}}}{\mathrm{M}_{\mathrm{Ti}_{2}}}\right. \\
& \left.-\frac{.027( \pm .006) \mathrm{W}_{\mathrm{M}_{\mathrm{g}} \mathrm{O}}}{\mathrm{M}_{\mathrm{Mg}_{\mathrm{g}}}}-\frac{.071( \pm .024) \mathrm{W}_{\mathrm{Ca} 0}}{\mathrm{M}_{\mathrm{Ca} 0}}\right)
\end{aligned}
$$

C) sedimentary silicate rocks, 20 samples, SEE $=.017$

$$
\begin{gathered}
P_{1}=\rho_{b}\left(\frac{.537( \pm .127) W_{A_{2} 0_{3}}}{M_{\mathrm{Al}_{2} O_{3}}}-\frac{.952( \pm .421) W_{K_{2} 0}}{M_{\mathrm{K}_{2} 0}}\right. \\
\left.-\frac{4.348( \pm 2.058) W_{\mathrm{T}: 0_{2}}}{M_{\mathrm{Ti}_{2}}}+\frac{1.407( \pm .685) \mathrm{W}_{\mathrm{M}_{\mathrm{g}} 0}}{\mathrm{M}_{\mathrm{Mg}_{\mathrm{g}} 0}}\right)
\end{gathered}
$$

The estimates of $P_{1}$ provided by ( 42 to 44 ) are found to be moderately well correlated with the values of $P_{1}$ determined for each of 56 samples $(r=.80)$ as shown in Figure 18 with a regression of

$$
P_{1}=1.007 P_{1 \text { calc }}
$$

For the frequency-dependent, conductive component $P_{2}$ of the loss factor, the stepwise linear regresion results for all silicate rock samples treated as a single class yield 


$$
\begin{gathered}
P_{2}=\rho_{b}\left(\frac{.117( \pm .04) W_{A_{2} O_{3}}}{M_{\mathrm{Al}_{2} O_{3}}}+\frac{.043( \pm .021) W_{\mathrm{LOI}}}{M_{\mathrm{LOI}}}\right. \\
\left.-\frac{3.447( \pm 2.349) \mathrm{W}_{\mathrm{PO}_{5}}}{\mathrm{M}_{\mathrm{PO}_{5}}}+\frac{.003( \pm .005) \mathrm{W}_{\mathrm{Si}_{2}}}{\mathrm{M}_{\mathrm{Si}_{2}}}\right)
\end{gathered}
$$

The oxides in (46) are listed in decreasing order of significance. Similar to $P_{1}$, the esteimates of $P_{2}$ given by the single class regression (46) are poorly correlated to the values of $P_{2}$ determined for each sample by (38) with $r=0.41$ for the 54 samples.

$$
P_{2}=0.009+0.863 P_{2 \text { calc. }}
$$

Regression analyses of $P_{2}$ seperately for each silicate class yields

a) volcanic silicate rocks, 20 samples, SEE $=.013$,

$$
\begin{aligned}
P_{2}=\rho_{b} & \frac{.232( \pm .081) W_{A_{2} O_{3}}}{M_{\mathrm{Al}_{2} \mathrm{O}_{3}}}-\frac{.216( \pm .116) \mathrm{W}_{\mathrm{LOI}}}{\mathrm{M}_{\mathrm{LOI}}} \\
& \left.-\frac{.299( \pm .18) \mathrm{W}_{\mathrm{Na}_{2} \mathrm{O}}}{\mathrm{M}_{\mathrm{Na}_{2} \mathrm{O}}}+\frac{.011( \pm .007) \mathrm{W}_{\mathrm{Si}_{2}}}{\mathrm{M}_{\mathrm{Si}_{2}}}\right)
\end{aligned}
$$


b) plutonic silicate rocks, 14 samples, SEE $=0.12$,

$$
\begin{gathered}
P_{2}=\rho_{b}\left(\frac{26.24( \pm 5.219) W_{\mathrm{LOI}}}{M_{\mathrm{LOI}}}-\frac{13.268( \pm 2.604) \mathrm{W}_{\mathrm{Na}_{2} \mathrm{O}}}{\mathrm{M}_{\mathrm{Na}_{2} \mathrm{O}}}\right. \\
\left.-\frac{.05( \pm .015) \mathrm{W}_{\mathrm{Si}_{2}}}{\mathrm{M}_{\mathrm{Si}_{2}}}+\frac{.055( \pm .039) \mathrm{W}_{\mathrm{Al}_{2} \mathrm{O}_{3}}}{\mathrm{M}_{\mathrm{Al}_{2} \mathrm{O}_{3}}}\right)
\end{gathered}
$$

c) sedimentary silicate rocks, 20 samples, $\mathrm{SEE}=0.15$,

$$
\begin{aligned}
P_{2}=\rho_{b} & \left(\frac{.087( \pm .024) W_{\mathrm{LOI}}}{M_{\mathrm{LOI}}}+\frac{1.098( \pm .264) \mathrm{W}_{\mathrm{Na}_{2} 0}}{\mathrm{M}_{\mathrm{Na}_{2} \mathrm{O}}}\right. \\
& \left.-\frac{1.565( \pm .707) \mathrm{W}_{\mathrm{M}_{\mathrm{g}} 0}}{\mathrm{M}_{\mathrm{Mg}_{\mathrm{O}}}}-\frac{.146( \pm .064) \mathrm{W}_{\mathrm{Ca} 0}}{\mathrm{M}_{\mathrm{Ca} 0}}\right)
\end{aligned}
$$

Also similar to the results found for $P_{1}$, the estimates of $P_{2}$ provided by each class treated seperately using (47) to (49) are moderately correlated with the values of $P_{2}$ determined for each sample using (38) with $r=0.75$. As shown by Figure 19,

$$
P_{2}=0.004+0.839 P_{2 \text { calc }}
$$


Not suprisingly, $\mathrm{LOI}$ and $\mathrm{Na}_{2} \mathrm{O}$ are generally the most significant determinants of the conductive component of the loss factor. The quantity of LOI is presumed to be largely determined by the bound water content of a sample.

The aggregate effect of using bulk rock chemistry to explain the measured variance in $\varepsilon^{\prime \prime}$ as a function of frequency via $(38)$ is shown in Figure 20. Linear

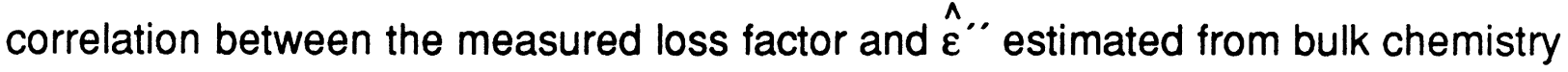
using estimates of $P_{1}$ from (42) to (44) and $P_{2}$ from (47) to (49) for each silicate class yields correlation coefficients in excess of 0.77 at each of the five frequencies. Thus, the empirical formulations generally account for better than $60 \%$ of the observed variance in $\varepsilon^{\prime \prime}$.

\subsection{CONCLUSIONS}

The real part of the permittivity $\varepsilon^{\prime}$ is found to be independent of frequency between $1.6 \mathrm{GHz}$ and $16 \mathrm{GHz}$. The bulk density of rock accounts for $50 \%$ of the observed variance in $\varepsilon^{\prime}$, and the geometric mean formulation adequately describes this relationship in confirmation with prior studies. For silicate rocks, the bulk chemical composition can account for an additional $28 \%$ of the variance in $\varepsilon^{\prime}$ provided that each silicate rock class (volcanic, plutonic, and sedimentary) are treated as distinct classes. This implies that mineralogical differences between the various silicate classes are significant with respect to $\varepsilon^{\prime}$. The most significant oxide constituents in determination of $\varepsilon^{\prime}$ are $\mathrm{SiO}_{2}, \mathrm{Fe}_{2} \mathrm{O}_{3}, \mathrm{~T}_{i} \mathrm{O}_{2}$, and $\mathrm{Na}_{2} \mathrm{O}$.

The dielectric loss factor $\varepsilon^{\prime \prime} \ll \varepsilon^{\prime}$. In general, $\varepsilon^{\prime \prime}$ decreases with frequency and can be modeled as the sum of a frequency-dependent conductive term $\mathrm{P}_{2}$ and a frequency-independent residual term $P_{1}$. The loss factor $\varepsilon^{\prime \prime}$ is very poorly correlated with the bulk density of rock as are the component terms $P_{1}$ and $P_{2}$. Bulk chemical composition as given by $\mathrm{x}$-ray fluorescence can account for $60 \%$ of the variance in $\varepsilon^{\prime \prime}$ at frequencies from $1.6 \mathrm{GHz}$ to $16 \mathrm{GHz}$ when silicates are subgrouped by genesis 
(i.e., volcanic, plutonic and sedimentary). Hence, mineralogy and crystal structure are presumed to be important determinants of dielectric loss. The measured loss is greatest for volcanic silicates and least for carbonates. For silicate rocks, the most important oxide consituents determining $\varepsilon^{\prime \prime}$ are $\mathrm{Fe}_{2} \mathrm{O}_{3}, \mathrm{TiO}_{2}, \mathrm{LOI}, \mathrm{Al}_{2} \mathrm{O}_{3}, \mathrm{Na}_{2} \mathrm{O}$, and $\mathrm{MgO}$. The significance of $\mathrm{LOI}$ is greatest for sedimentary silicates and least for plutonic silicates, and may be related to the role of bound water in influencing dielectric loss.

The empirical relationships derived in this study yield highly satisfactory estimates of permittivity as functions of bulk density and bulk chemical composition for the frequency range from $1.6 \mathrm{GHz}$ to $16 \mathrm{GHz}$. In applying these results to a terrestrial evnironment, the role of both adsorbed and absorbed water must be kept in mind as this will influence both $\varepsilon^{\prime}$ and the loss factor. Finally, in light of these results it is noted that the role of mineralogy and crystal structure remains to be examined analytically. 


\section{REFERENCES}

1. Campbell, M.J. and J. Ulrichs, "Electrical properties of rocks and their significance for lunar radar observations," Journal of Geophysical Res., Vol. 74, pp. 5867-5881, 1969.

2. Olhoeft, G.R., A.L. Frisillo, and D.W. Strangway, "Electrical properties of lunar soil sample 15301, 38," Journal of Geophysical Res. Vol. 79, pp. 1599-1604, 1974.

3. Strangway, D.W., G.R. Olhoeft, W.B. Chapman, and J. Carnes, "Electrical properties of lunar soils: dependence upon frequency, temperature and moisture," Earth Planet Sci. Lett., Vol. 16, pp. 275-281, 1972.

4. Olhoeft, G.R. and D.W. Strangway, "Dielectric properties of the first 100 meters of the moon," Earth Planet Sci. Lett., Vol. 24, pp. 394-404, 1975.

5. Parkhomenko, E.I., Electrical Properties of Rocks, trans. G.V. Keller, Plenum Press, New York, pp. 314, 1967.

6. von Hippel, A.R., Dielectric Materials and Applications," John Wiley, New York, pp. $405,1954$.

7. Athey, T.W., M.A. Stuchly, and S.S. Stuchly, "Measurement of radio frequency permittivity of biological tissues with an open-ended coaxial line: Part I," IEEE Trans. Microwave Theory Tech., vol. MTT-30, no. 1, pp. 82-86, Jan. 1982.

8. Stuchly, M.A., M.M. Brady, S.S. Stuchly, and G. Gajda, "Equivalent circuit of an open-ended coaxial line in a lossy dielectric," IEEE Trans. on Instrumentation and Meas., Vol. IM-31, No. 2, June, 1982.

9. Burdette, E.C., F.L. Cain, and J. Seals, "In Vivo probe measurement technique for determining dielectric properties at VHF through microwave frequencies," IEEE Trans. Microwave Theory Tech, vol. MTT-28, no. 4, p. 414-427, April, 1980.

10. El-Rayes, M.A., and F.T. Ulaby, "Microwave dielectric spectrum of vegetation Part 1: experimental observations," IEEE Trans. Geoscience and Remote Sensing, Vol. GE-25, No. 5, pp. 550-557, 1987.

11. Marcuvitz, N., Wavequide Handbook, New York, Dover, 1965.

12. Deschamps, G.A., "Impedance of antenna in a conducting medium," IRE Trans. Antennas Propagat., pp. 648-650, Sept. 1962. 
13. Ulaby, F.T., R.K. Moore, and A.K. Fung, Microwave Remote Sensing, vol. 3, Dedham, MA: Artech, 1986, Appendix E.

14. Jordan, B.P., R.J. Sheppard, and S. Szwarnowski, "The dielectric properties of formamide, ethanediol, and methanol," J. Phys. D: Appl. Phys., vol. 11, pp. 695$701,1978$.

15. Harrington, Roger F., Time-Harmonic Electromagnetic Fields, New York, McGraw-Hill Book Company, pp. 317-326, 1961.

16. Ulaby, F.T., T. Bengal, J. East, M.C. Dobson, J. Garvin, and D. Evans, "Microwave dielectric spectrum of rocks," Radiation Lab. Tech. Rep. No. 023817-1-T, Department of Electrical Eng. and Computer Sci., Univ. of Michigan, Ann Arbor, March, 1988. 


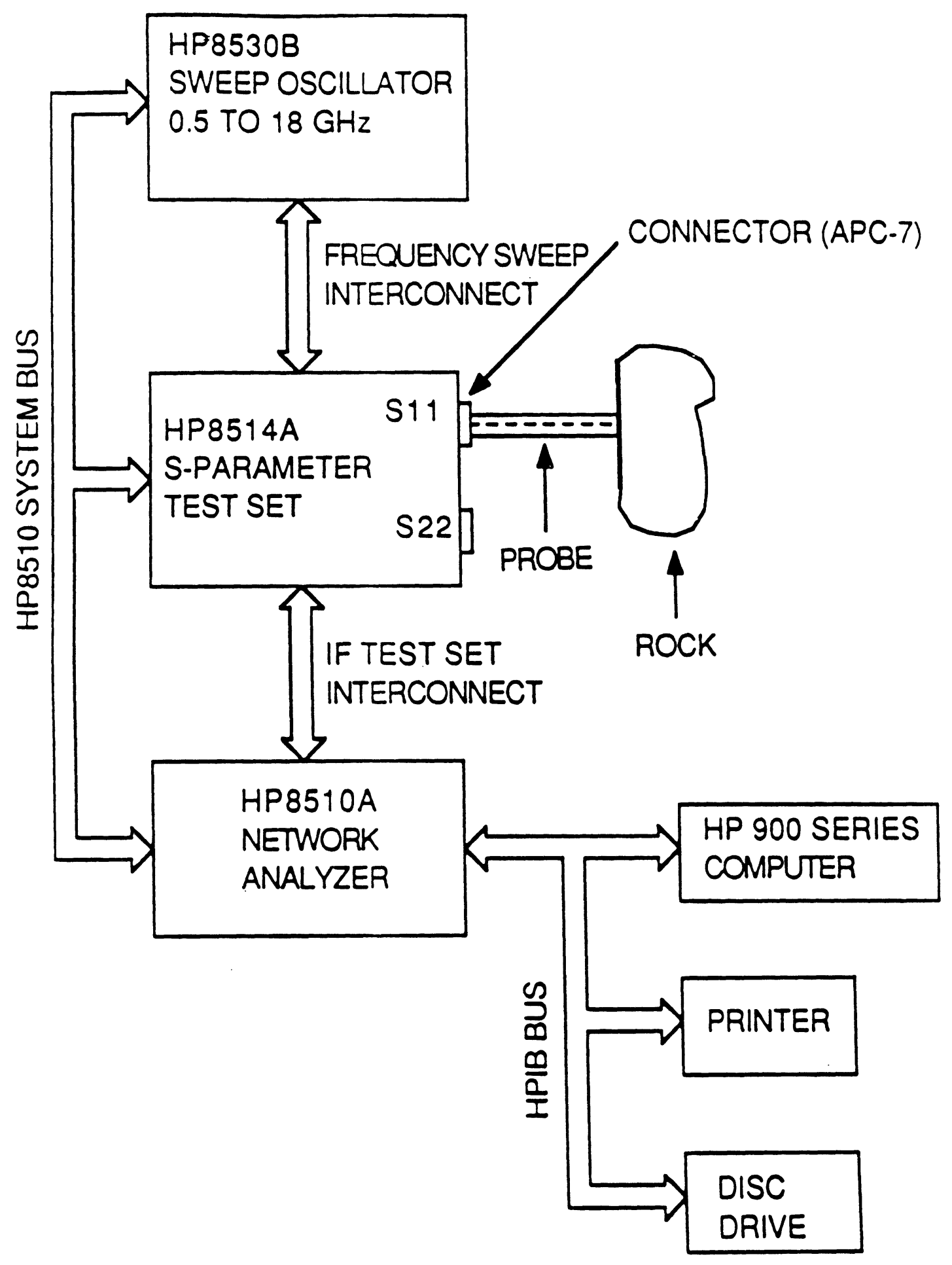

Fig. 1. Block diagram of the dielectric-probe measurement system. 


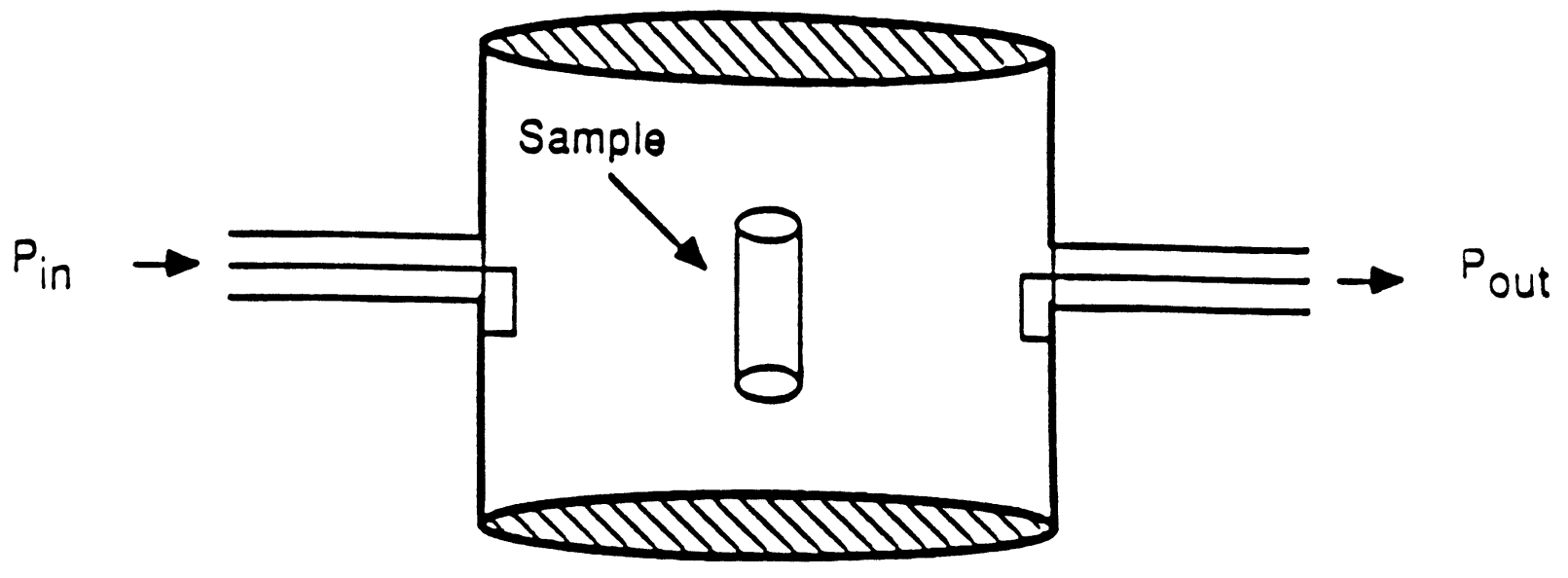

Resonant Cavity

(a) Cavity with Sample

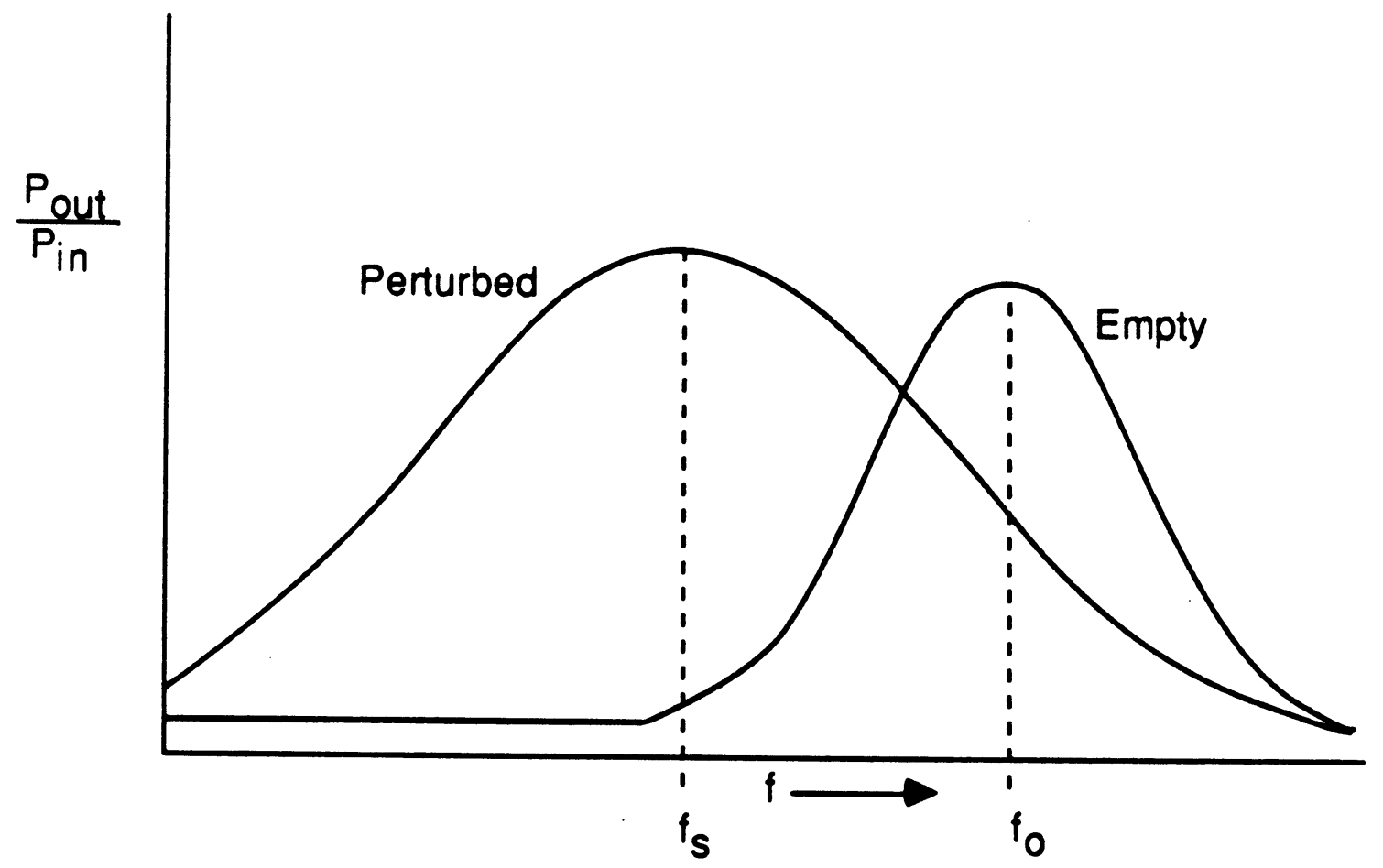

(b) Transmission Spectra

Figure 4. When a sample is inserted in the cavity, its transmission spectrum changes: the resonant frequency shifts from $f_{0}$ to $f_{s}$ and the spectrum becomes broader. 


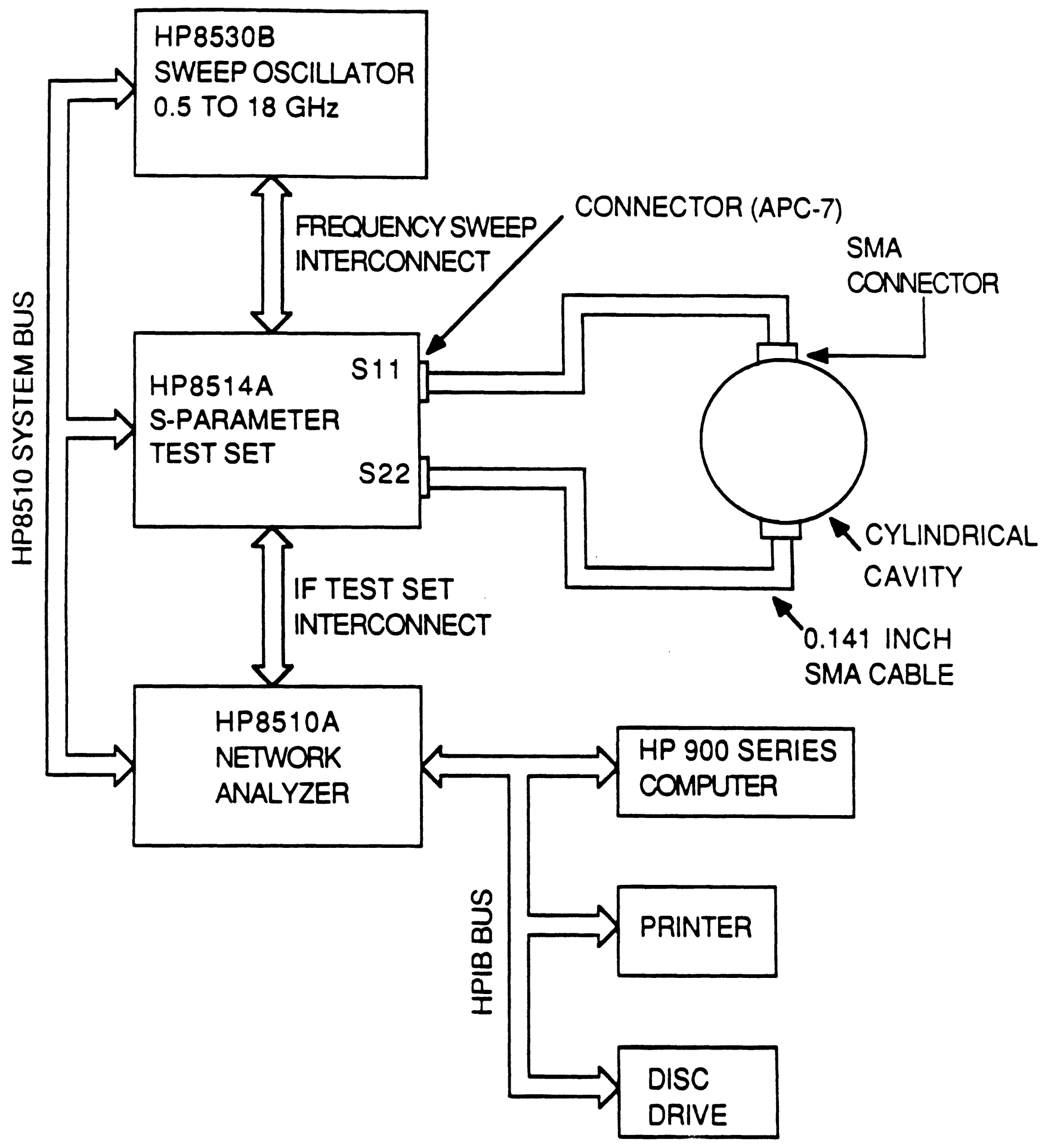

Figure 5. Block diagram of the system used to measure the cavity transmission spectrum. 


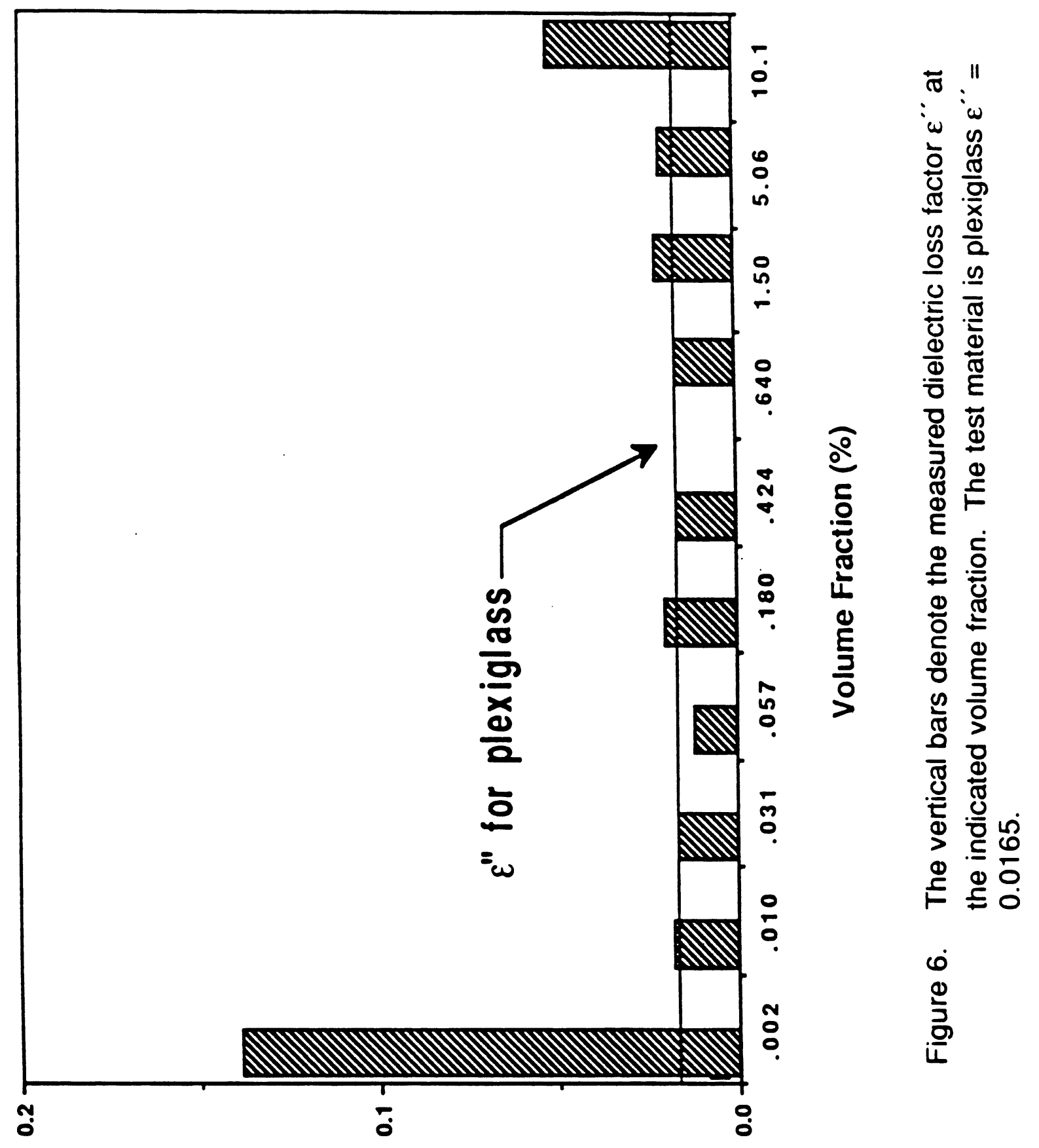

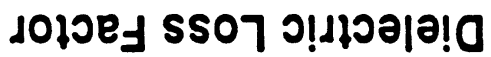




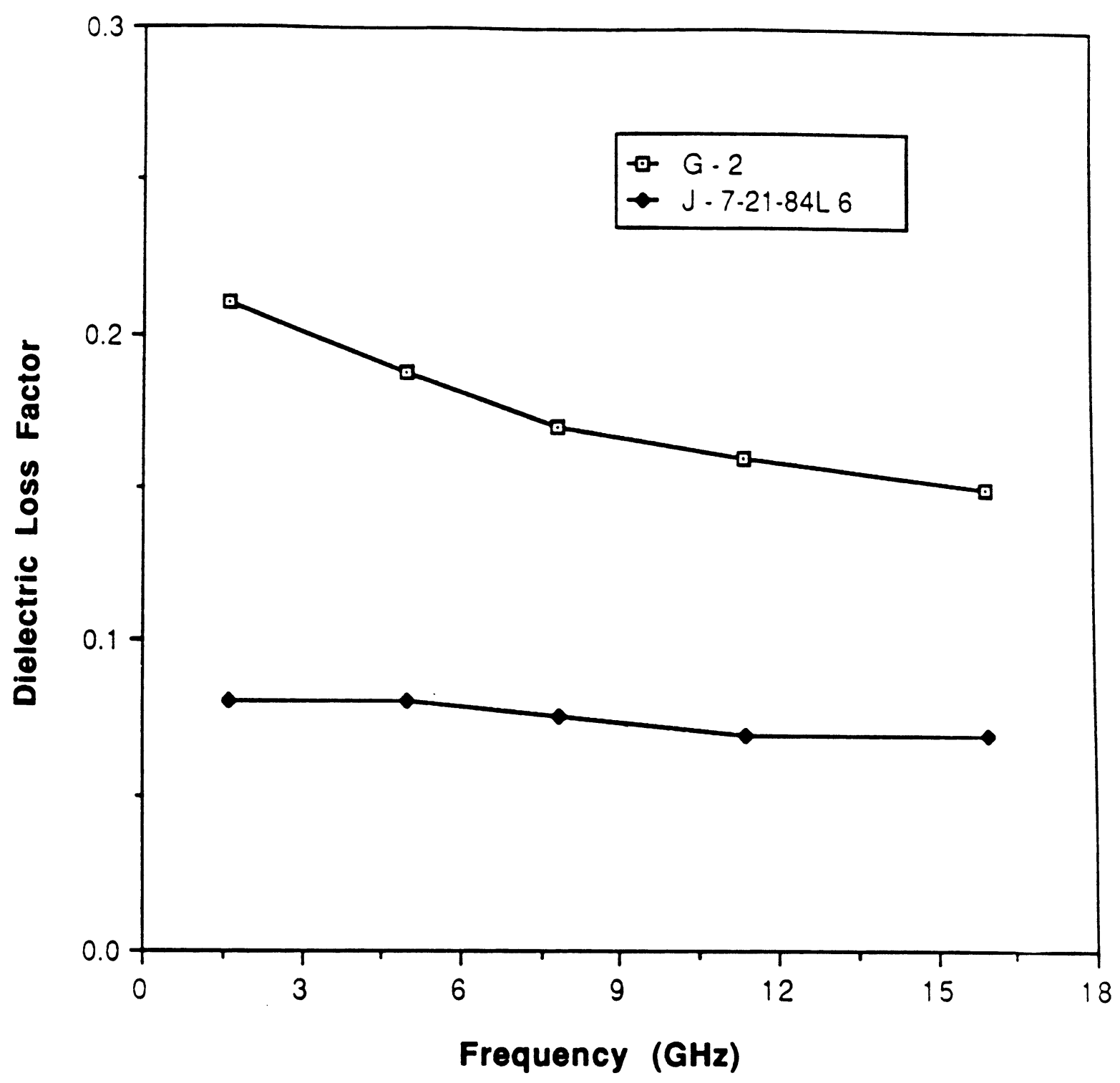

Figure 7. Typical examples of the measured spectra of the dielectric loss factor $\varepsilon^{\prime \prime}$. 


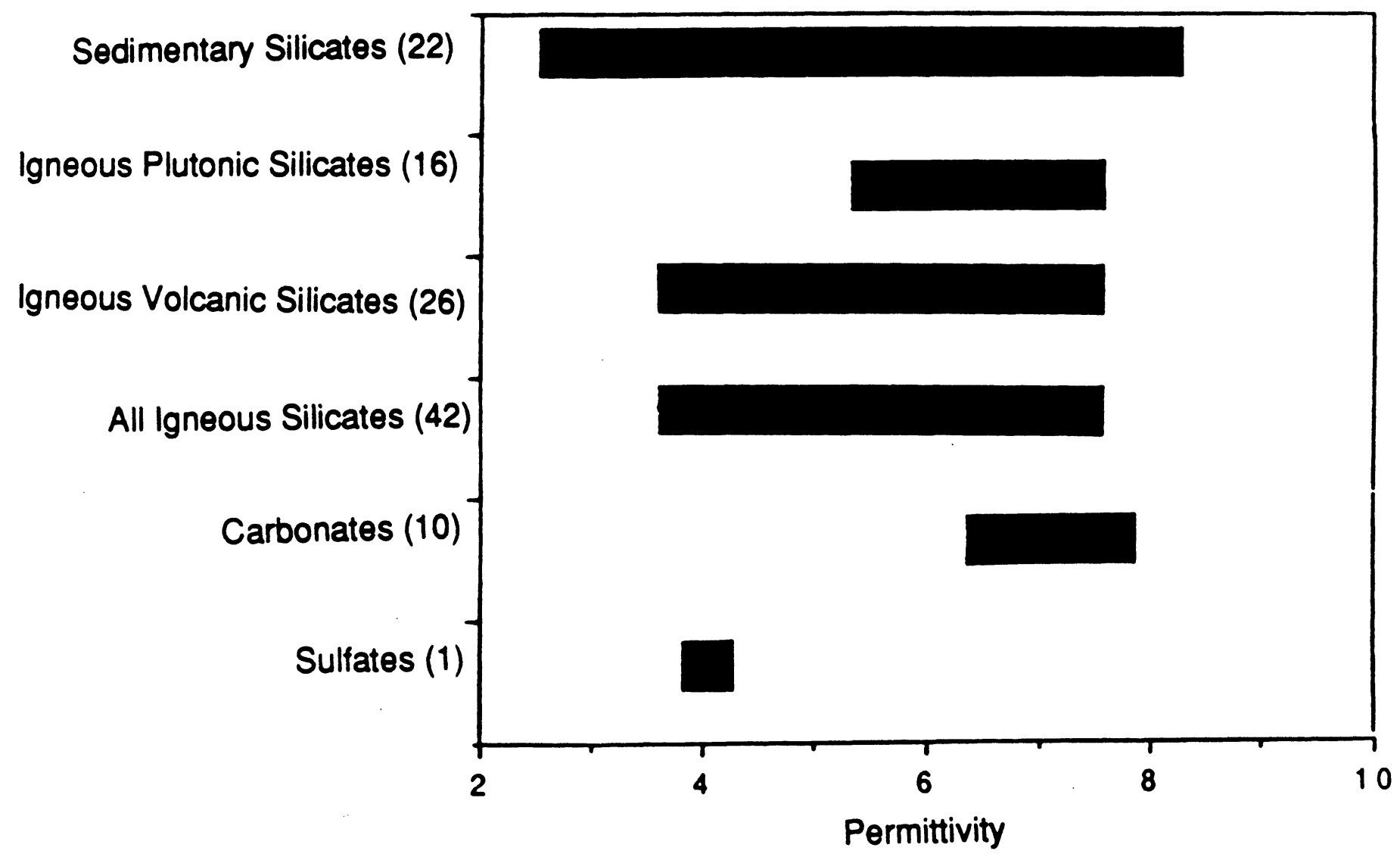

Figure 8. Range of $\varepsilon^{\prime}$ for individual rock classes. 


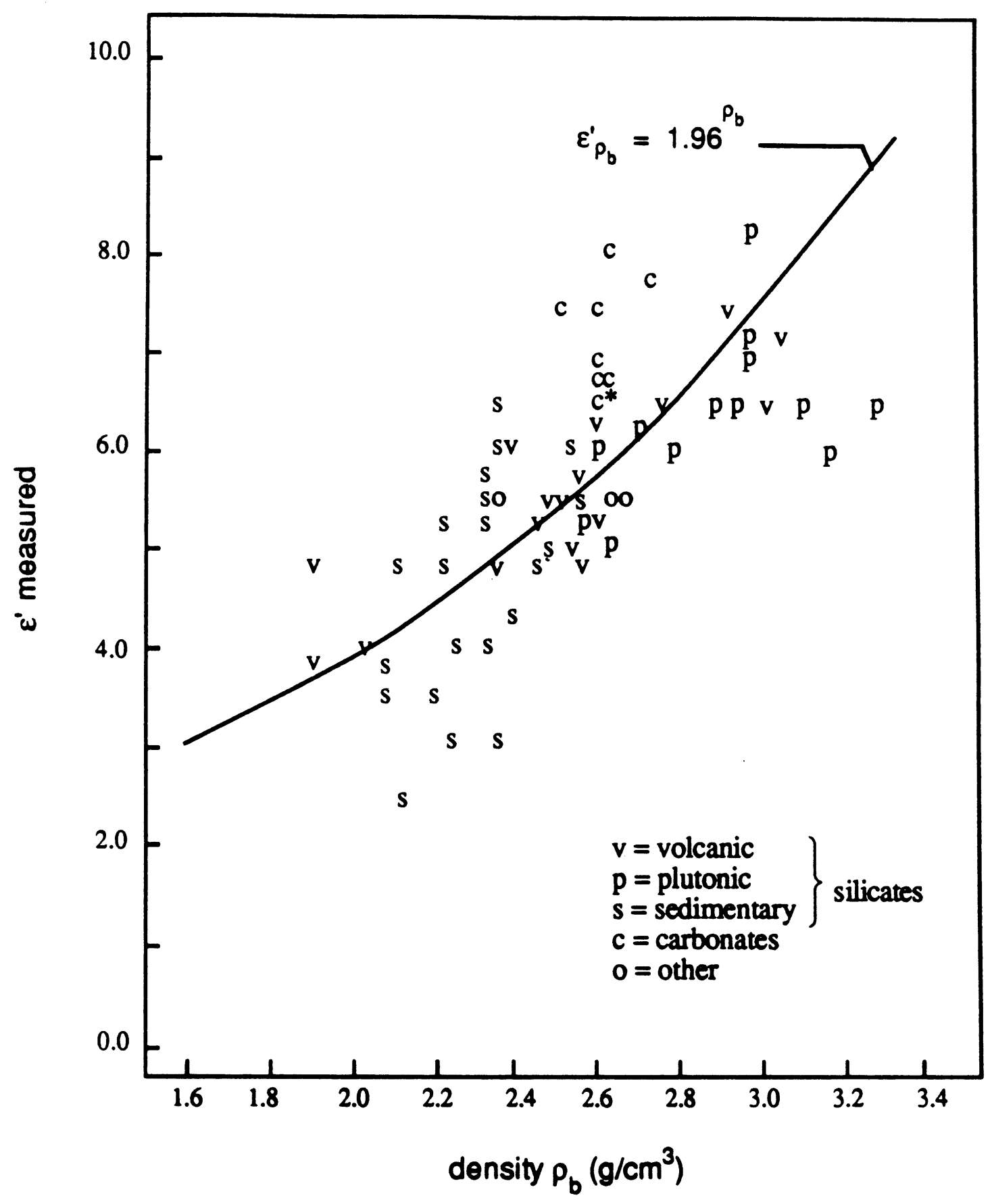

Figure 9. Variation of the real part of the dielectric constant $\varepsilon^{\prime}$ with rock bulk density. 


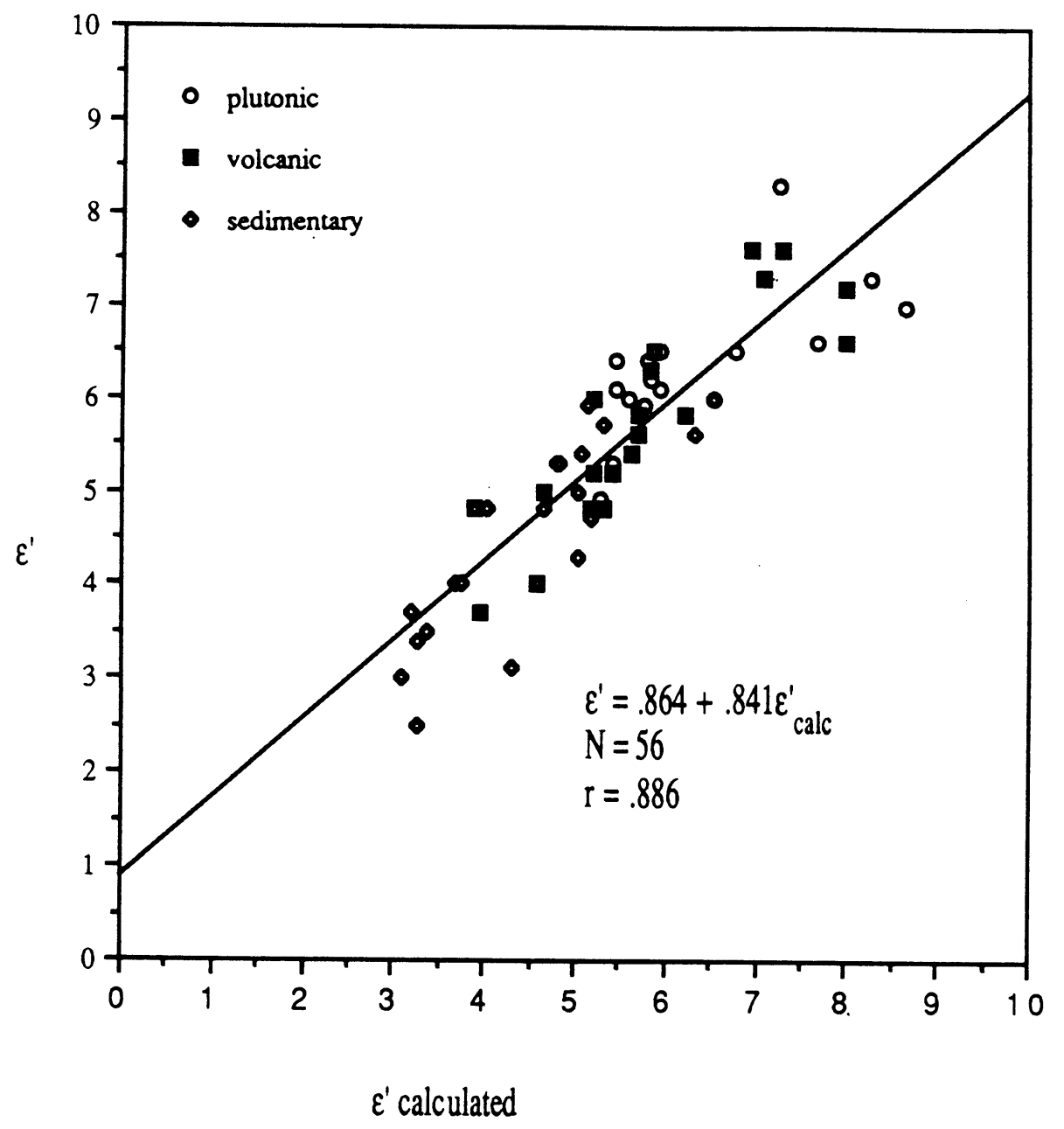

Figure 10. Comparison of measured $\varepsilon^{\prime}$ with the permittivity estimated on the basis of density and bulk chemistry by (35) to (37) for volcanic, plutonic and sedimentary silicate rocks. 


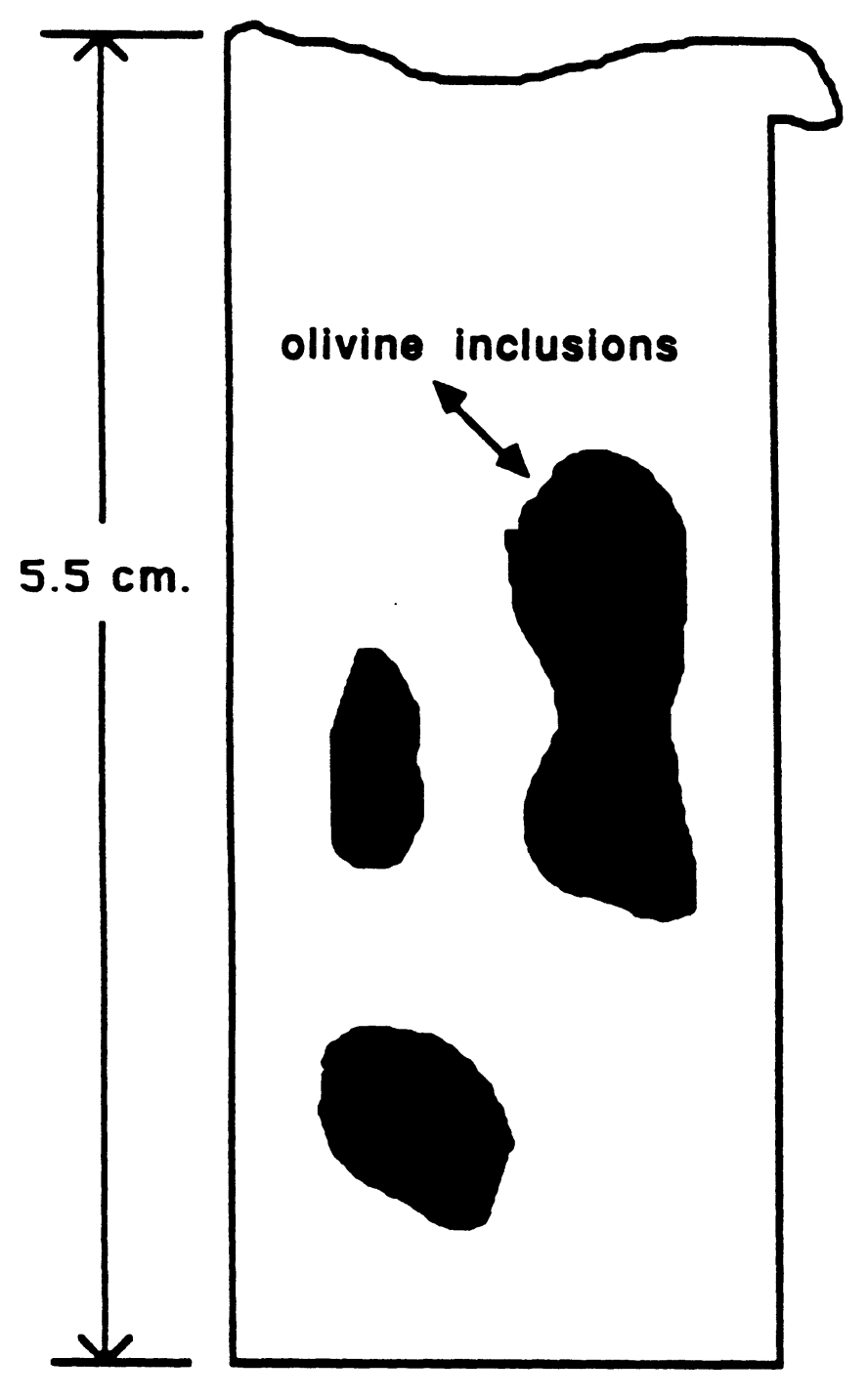

(a) Sketch of rock surface

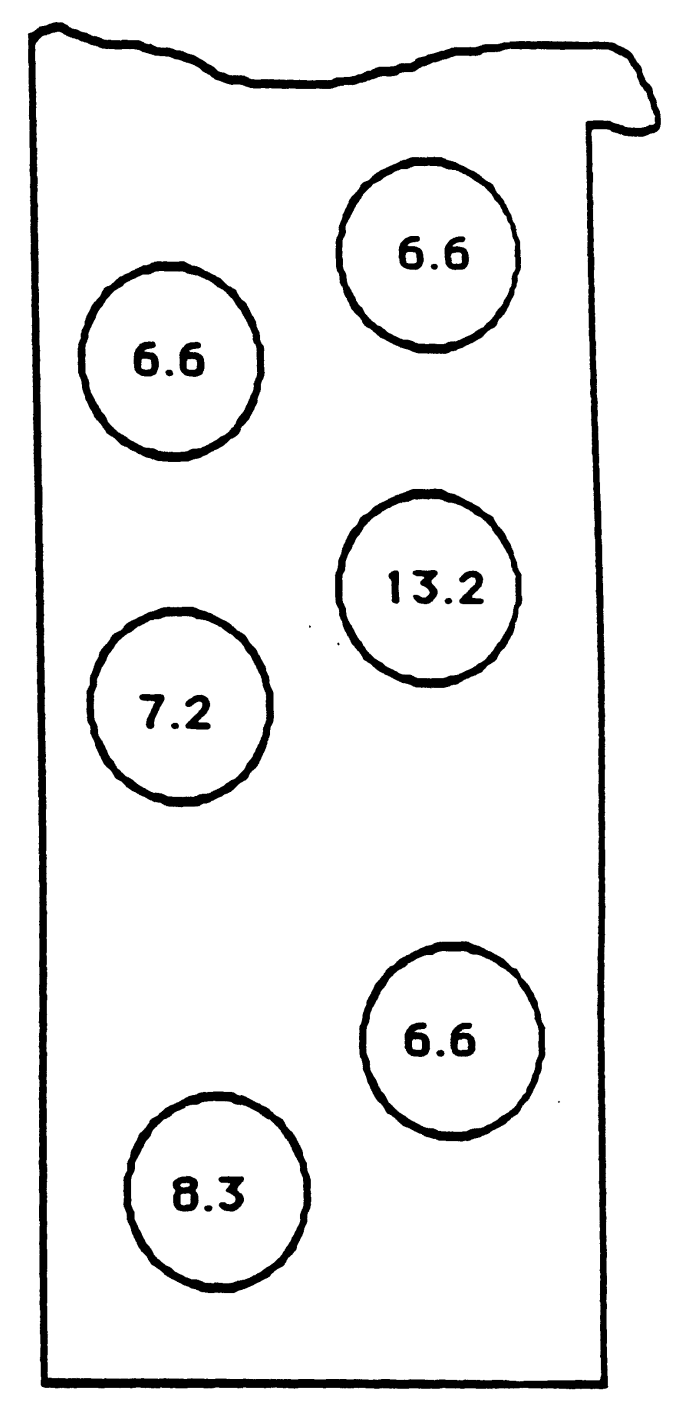

(b) Measured permittivity

Figure 11. Effect of mineral inclusions on $\varepsilon^{\prime}$ for a sample of gabbro. 


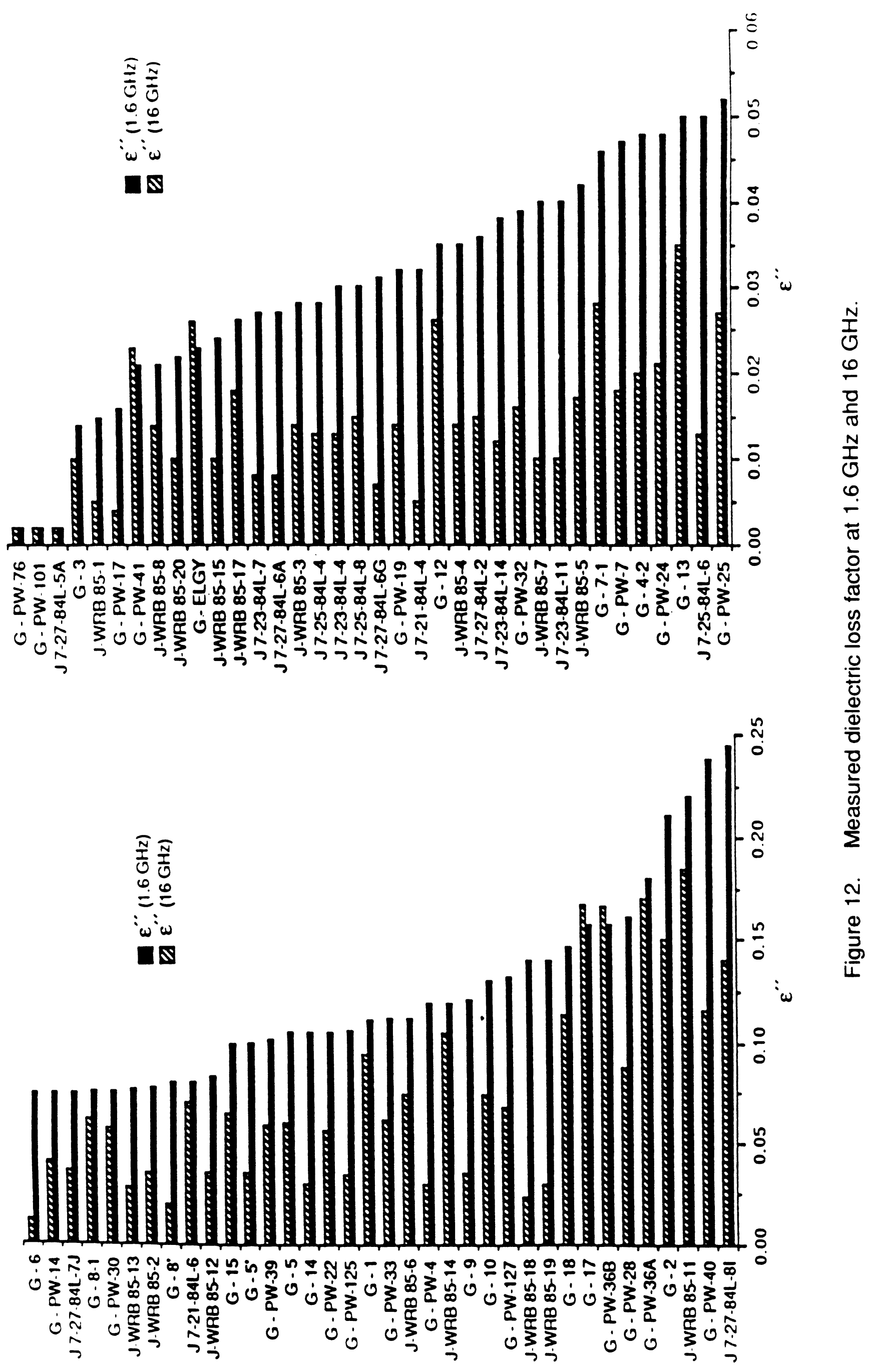




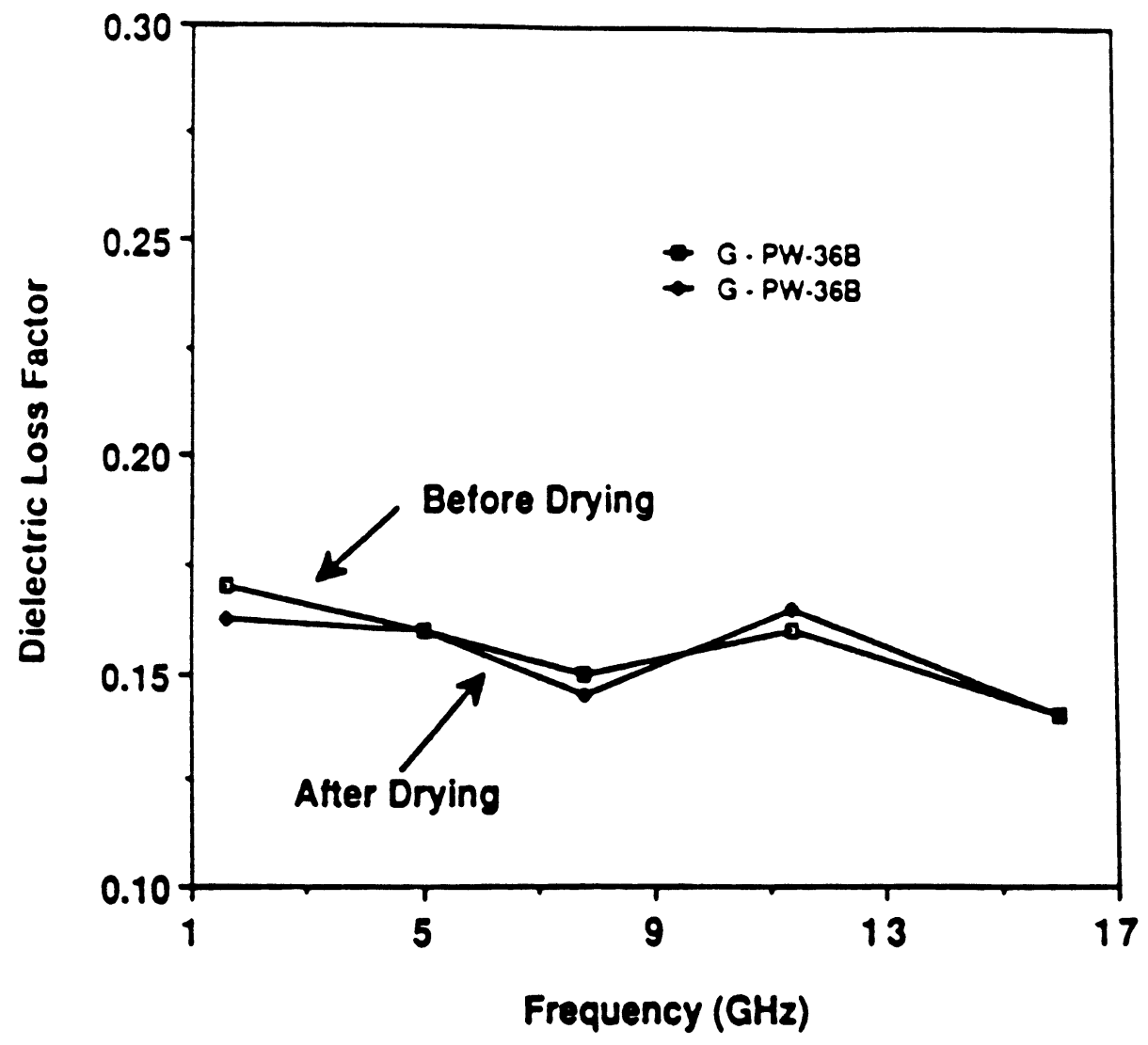

(a) Sample PW-36B (basalt)

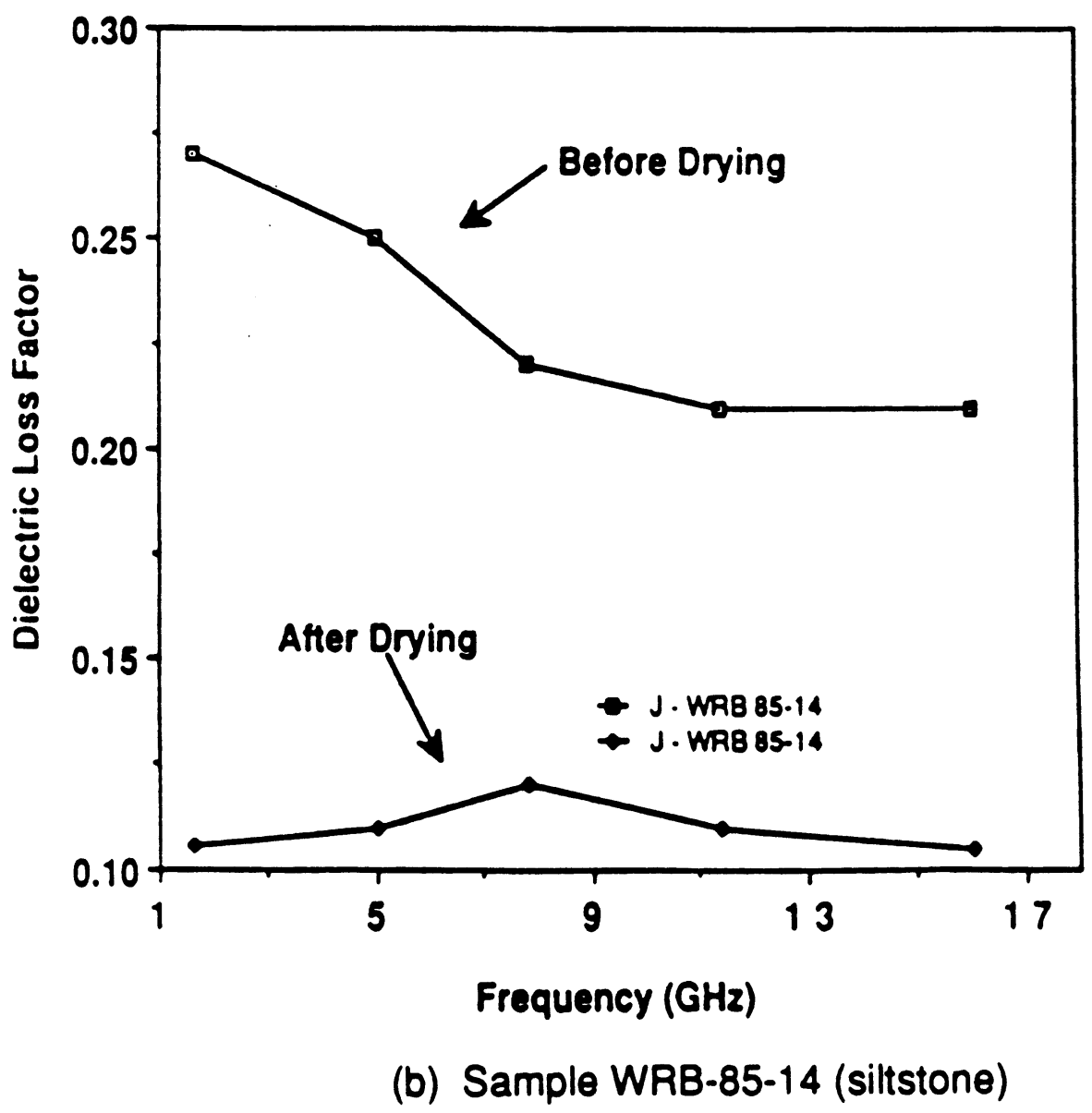

Figure 13. Effect of drying surficicial water on $\varepsilon^{\prime \prime}$. 


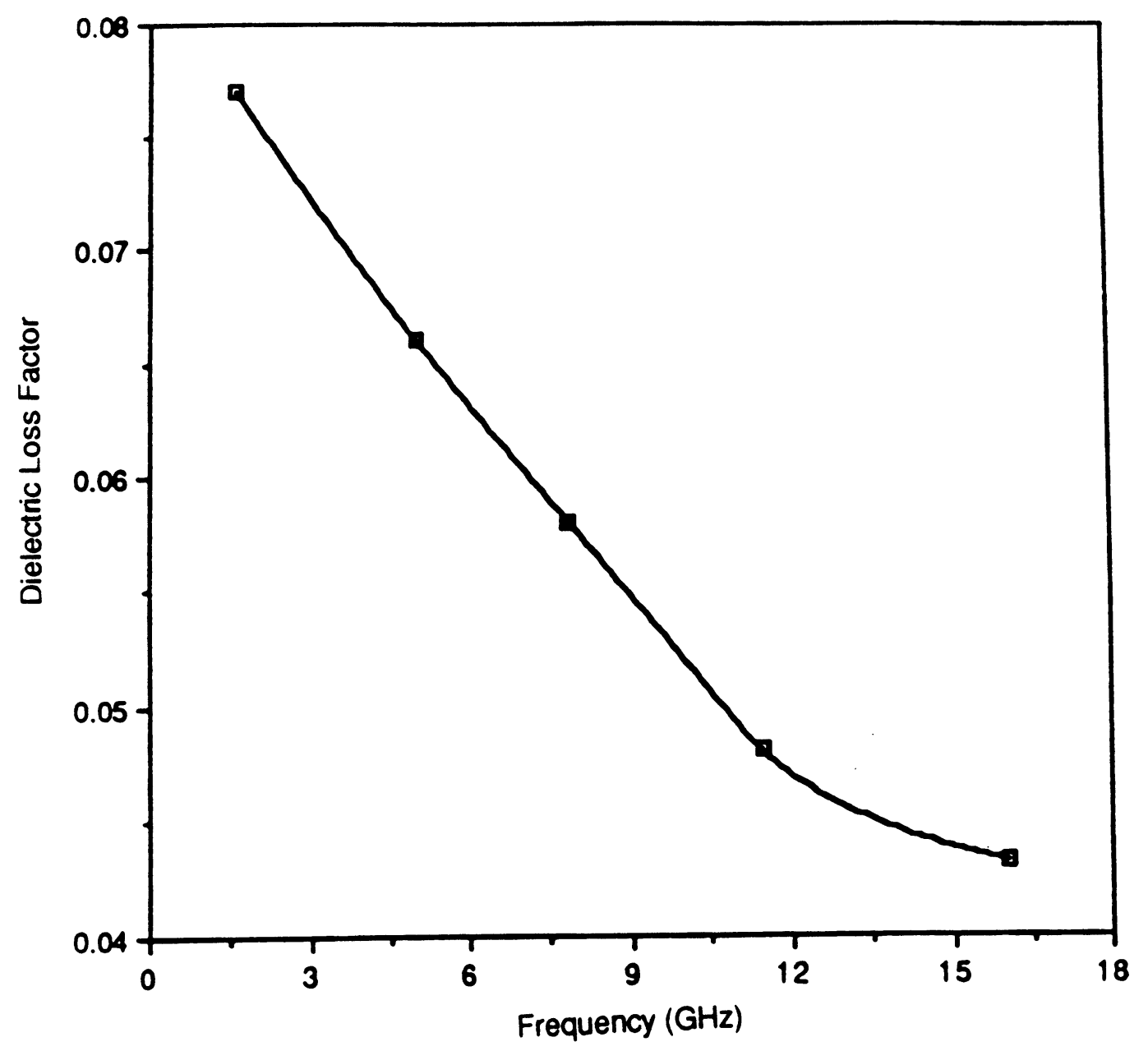

Figure 14. Average dielectric loss factor of all 72 rock samples versus frequency. 


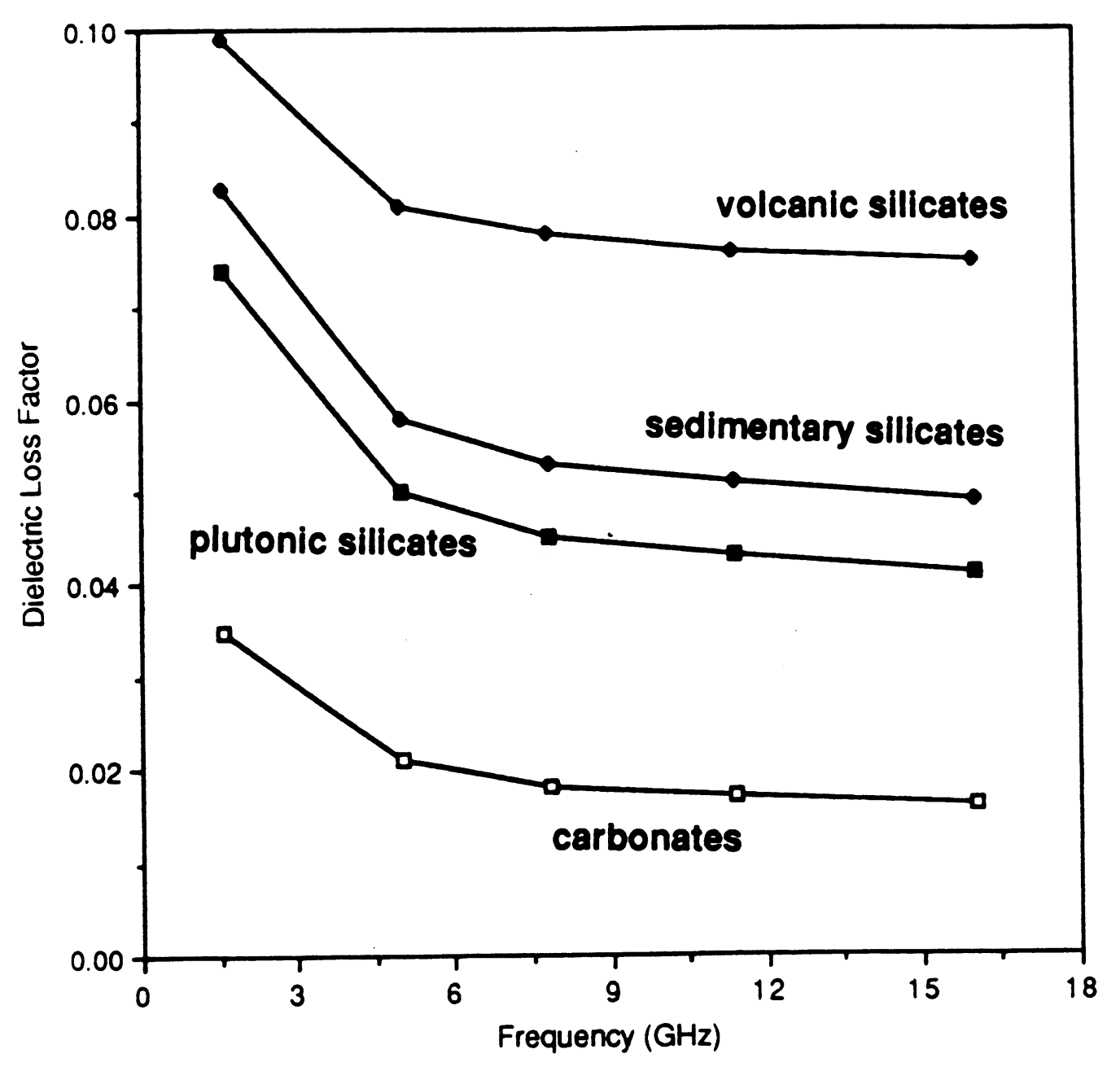

Figure 15. Variation in average loss spectrum for various rock classes. 
$a^{N}$

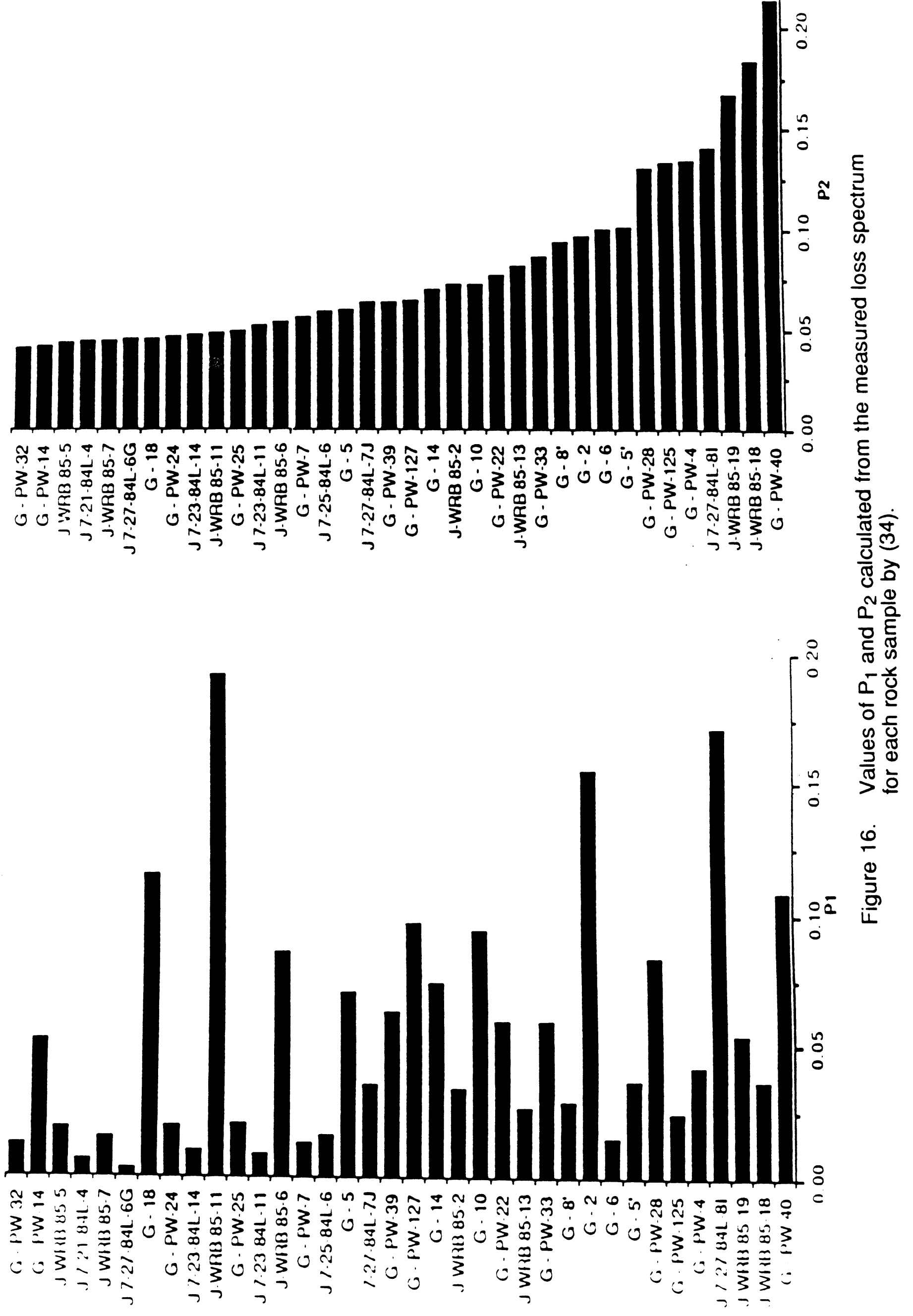



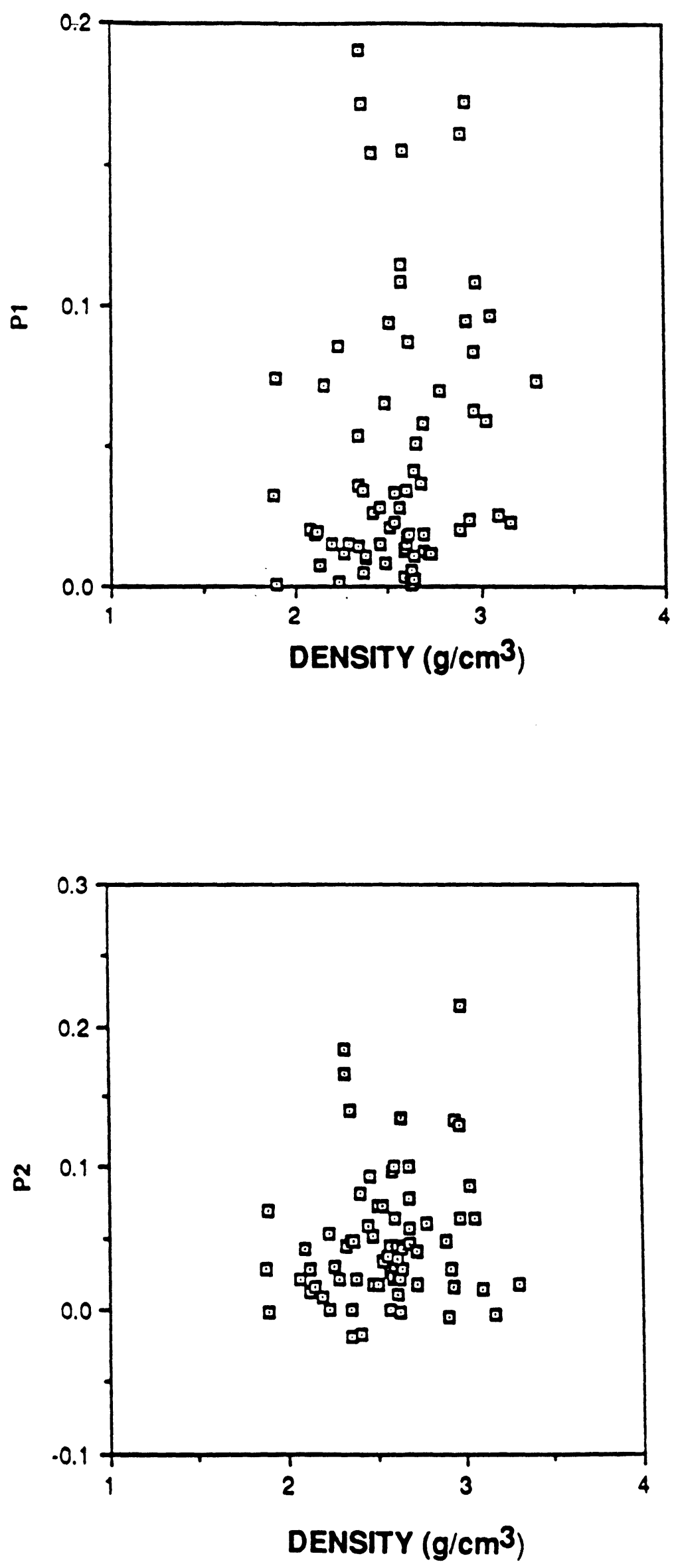

Figure 17. Variation of $P_{1}$ and $P_{2}$ as calculated by (34) with rock density. 


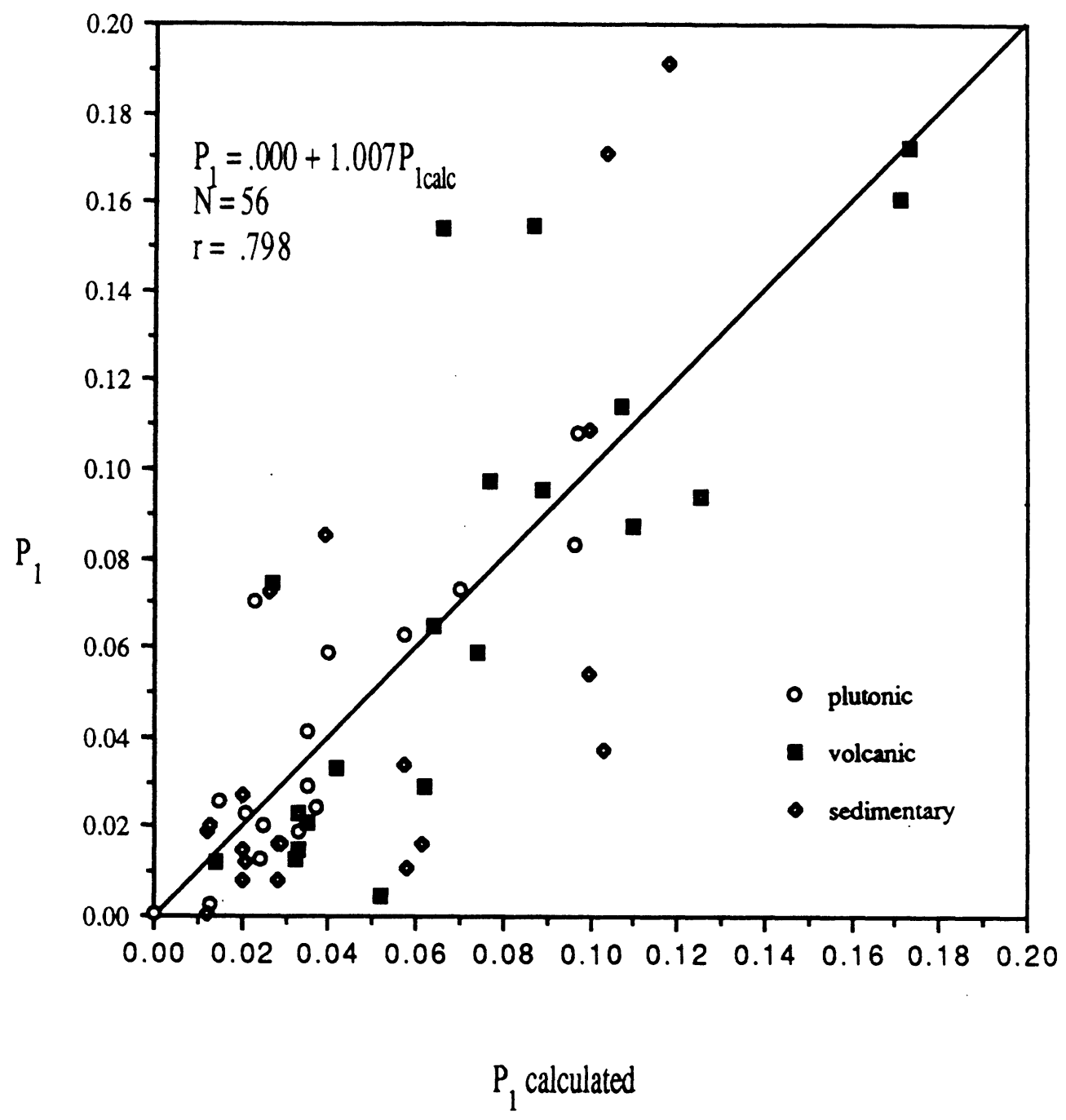

Figure 18. Comparison of observed $P_{1}$ with that estimated on the basis of density and bulk chemistry for each silicate rock class (volcanic, plutonic and sedimentary) using (42) to (44), respectively. 


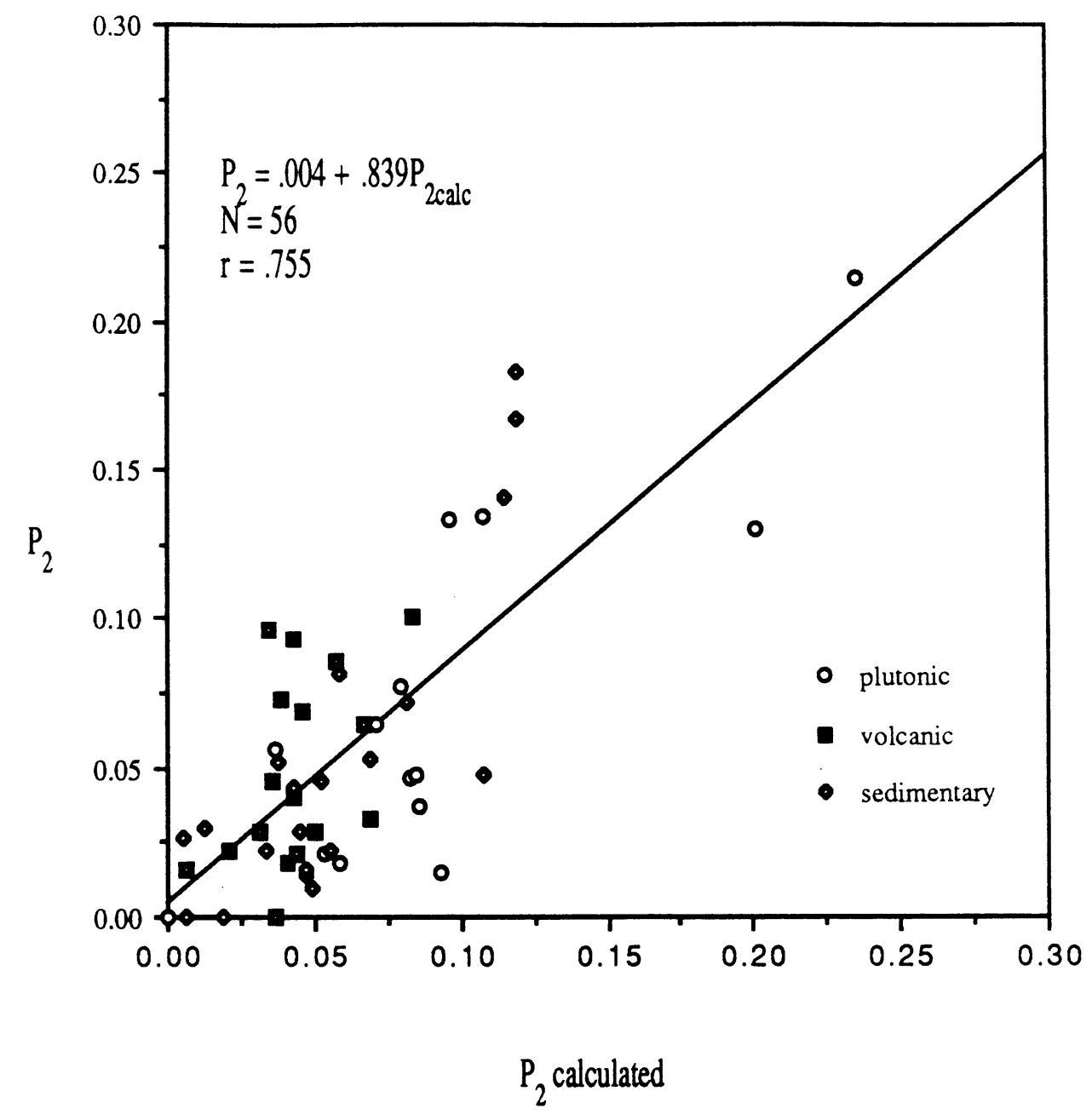

Figure 19. Comparison of observed $\mathrm{P}_{2}$ with that estimated on the basis of density and bulk chemistry for each silicate rock class (volcanic, plutonic and sedimentary) using (47) to (49) respectively. 


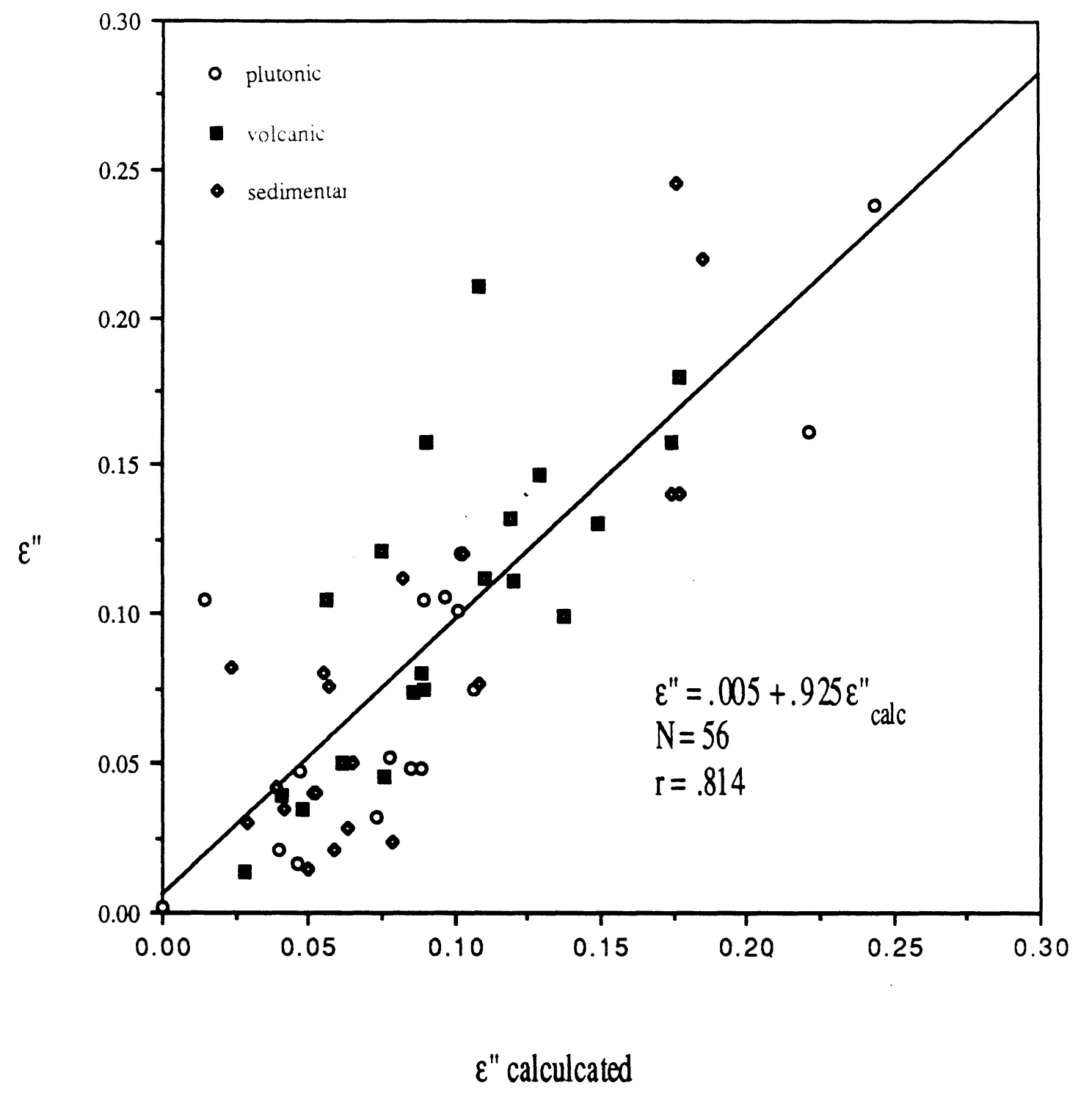

a) Frequency $=1.6 \mathrm{GHz}$

Figure 20. Relationships of measured loss factor to the loss factor estimated on the basis of density and bulk chemistry at (a) $1.6 \mathrm{GHz}$, (b) $7.8 \mathrm{GHz}$, and (c) $16 \mathrm{GHz}$. 


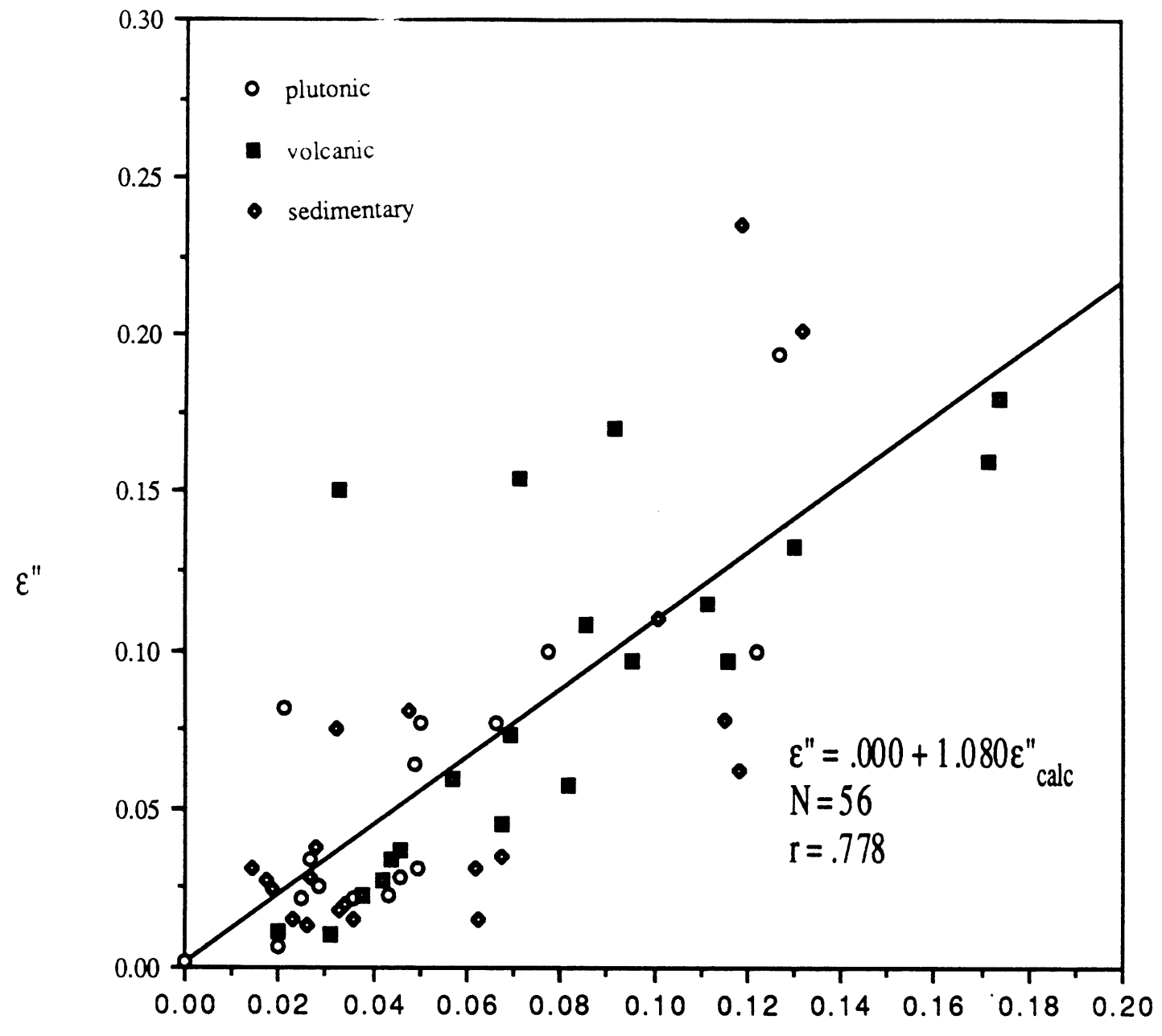

$\varepsilon "$ calculated

b) Frequency $=7.8 \mathrm{GHz}$ 


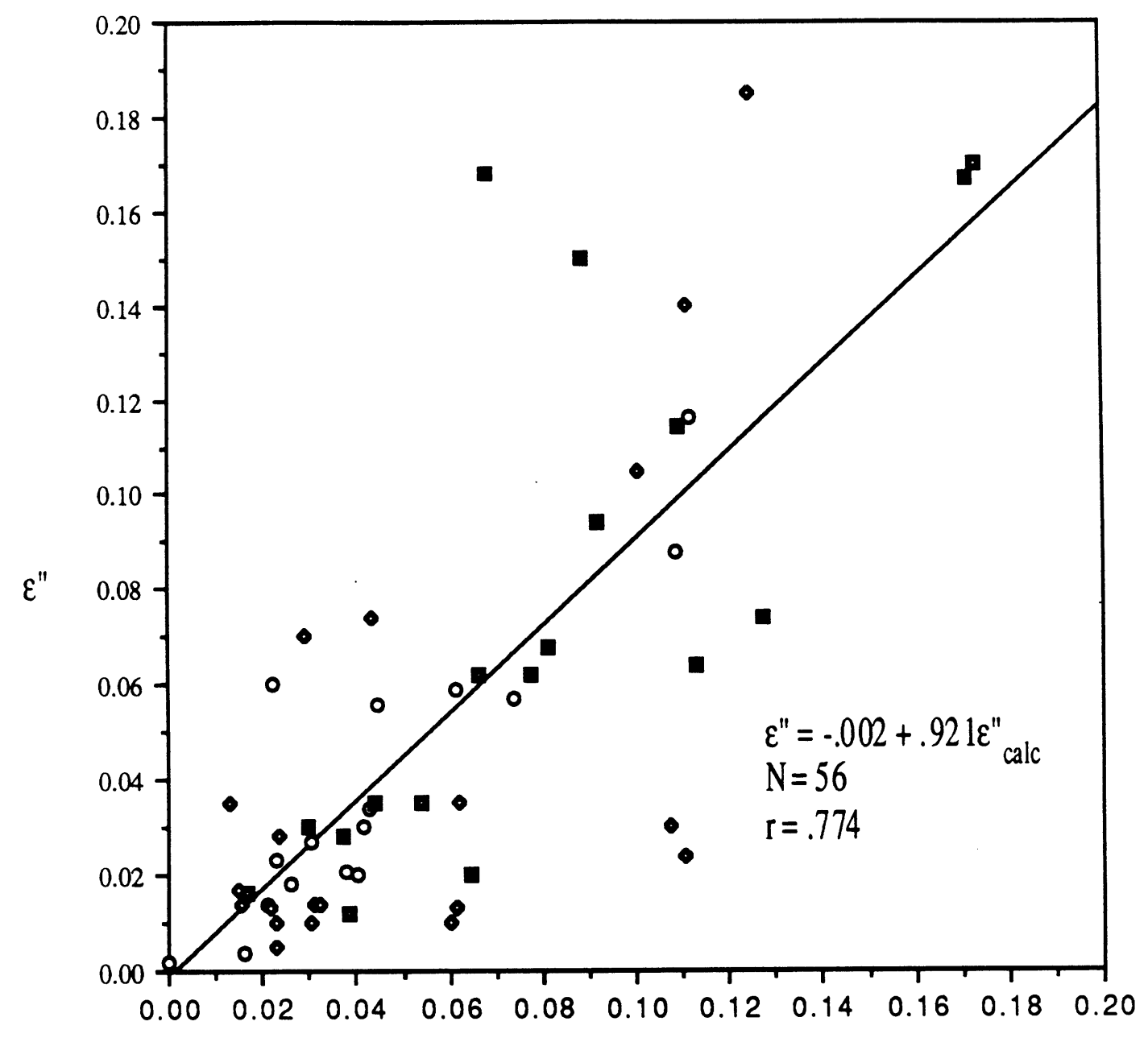

$\varepsilon^{\prime \prime}$ calculated

c) Frequency $=16 \mathrm{GHz}$ 